Bull. Geol. Soc. Finland 41, 21-45 (1969)

\title{
INITIALER MAGMATISMUS UND SEINE ERZBILDUNG IN DER BELEUCHTUNG DES KOLI-KALTIMOGEBIETS
}

\author{
Tauno Pitrainen \\ Institut für Geologie, Universität Oulu, Finnland
}

\begin{abstract}
In the Koli-Kaltimo area, North Karelia, Finland, the initial igneous activity of the Karelidic zone has produced diabase dikes and spilitic sills. Some of the sills have differentiated forming spilitic rock associations, which range from peridotite to albite diabase rich in albite. The discussion of the spilites and the ore forming processes in the crystallisation of spilites is based on field observations, microscopical investigation, the geochemical distribution of some metals in the rocks and minerals, and the isotope ratio of sulphur. Both the diabase dikes and the spilitic sills have originated from the same tholeitic parent magma, the diabases from the »dry» magma and the spilites from the magma that has been rich in water and carbon dioxide. Also the ore-forming processes are different in these two magmas.
\end{abstract}

\section{INHALTSVERZEICHNIS}

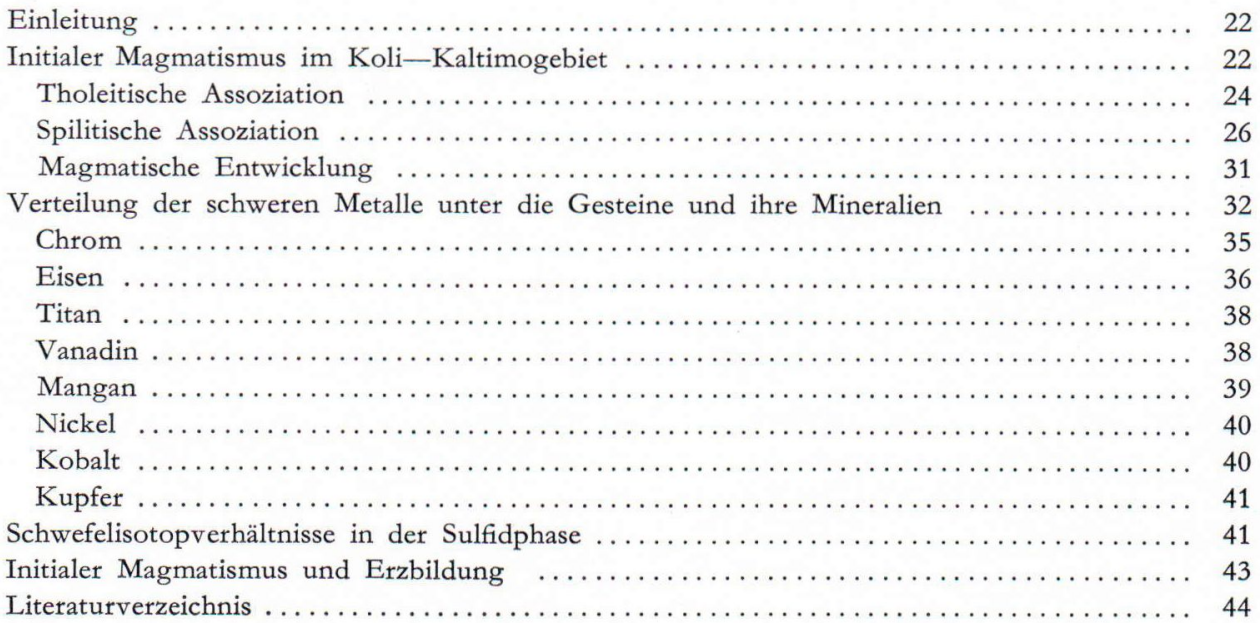




\section{Einleitung}

Diese Arbeit ist der erste Teil einer grösseren Untersuchung »Initialer Magmatismus und seine Erzbildung in der karelischen Zone», welche am geologischen Institut der Universität Oulu durchgeführt wird und hier im Lichte des KoliKaltimogebiets mit Rücksicht auf geochemische Untersuchungen betrachtet wird. Den ersten Teil des Themas, den initialen Magmatismus, habe ich (1968) schon in meiner Untersuchung über die Uranerzlagerstätte des Gebiets behandelt. Darauf habe ich im Sommer 1967 meine frühere Probensammlung von den betreffenden Gesteinen für die Untersuchung der Erzbildung, den zweiten Teil des Themas, ergänzt. Die Erzbildung wird hier mit Rücksicht auf die Verteilung der wichtigsten schweren Metalle unter die verschieden Gesteinsarten und ihre Mineralien erklärt.

Mikroskopische Untersuchungen für diese Arbeit habe ich am geologischen Institut der Universität Oulu gemacht. Dem Leiter der Anstalt, Herrn Professor Juhani Seitsaari, sage ich meinen besten Dank dafür, dass ich an dem von ihm geleiteten Institut habe arbeiten dürfen, sowie auch für die Unterstützung, die ich während der Arbeit bekommen habe.

Die chemischen Analysen, mit deren Hilfe die Verteilung der schweren Metalle unter die verschiedenen Gesteinsarten beleuchtet worden ist, sind im Labor von Geologinen tutkimuslaitos gemacht worden, wofür ich dem Generaldirektor, Herrn Professor Vladi Marmo und dem Leiter der chemischen Abteilung, Herrn Professor Eetu Savolainen, meinen besten Dank sagen will. Die Elektronenmikrosondebestimmungen, die darauf hinweisen, wie die schweren Metalle unter die verschiedenen Mineralien in den betreffenden Gesteinsarten verteilt sind, sind im Labor der Outokumpu A.G. gemacht worden, wofür ich dem Leiter der Erzsuche, Herrn Magister Pauli Isokangas und dem Leiter des Labors, Herrn Doktor Aulis Häkli sehr danken möchte. Zum Schluss danke ich besonders Herrn Uni- versitätslektor Rolf Ehnert für die sprachliche Korrektur des Manuskripts und Stud. Maija Outakoski für die Zeichnung der Abbildungen.

\section{Initialer Magmatismus im Koli-Kaltimo- gebiet}

Wie es in der geologischen Karte des KoliKaltimogebiets (s. Abb. 1) zu sehen ist, haben sich die basischen Erstarrungsgesteine im Gebiet sehr weit verbreitet. Sie sind schon früher von Frosterus (1902), Frosterus \& Wilkman (1920), Väyrynen (1939, 1954), Gaál (1965) und Piirainen (1968) behandelt worden. Frosterus und Frosterus \& Wilkman haben in ihnen zwei Gruppen, Metabasite und Uralitdiabase unterschieden. Nach ihnen sind die Metabasite ältere, stark metamorphosierte basische Gänge, die nur die kalevische Formation durchschneiden, und die Uralitdiabase sird jüngere, schwach metamorphosierte basische Gänge, die sowohl die kalevische als auch die jatulische Formation durchschneiden. Nachdem das Kalev und das Jatul ihren ursprünglichen Sinn verloren und eine neue Bedeutung als selbständige Formationen in der karelischen Orogenese bekommen haben, und zwar so, dass man festgestellt hat, dass das Kalev jünger als das Jatul ist (Väyrynen 1928, Wegman 1928), hat die Einteilung in die kalevischen Metabasite und in die jatulischen Uralitdiabase keine Bedeutung mehr. Väyrynen (1933) spricht nur von Metabasiten, die nach ihm älter als die kalevische Formation sind.

Neulich hat Gaál (1964) das Gebiet von neuem kartiert. Bei der Kartierung hat er das Hauptgewicht auf das Metapsephit-Metapsammitschichtenpaket und auf die Tektonik gelegt. Die Magmatite des Gebiets hat er dagegen nicht näher untersucht. Auf Grund seiner Auffassung, dass der obere Teil des Metapsephit-Metapsammitschichtenpakets eine karelische Molassebildung sei, deutet Gaál die hypabyssischen Erstarrungsgesteine als postorogene Diabase. Da die entsprechenden Vulkanite in der karelischen Schieferzone auf der evolutionärtransgressiven 


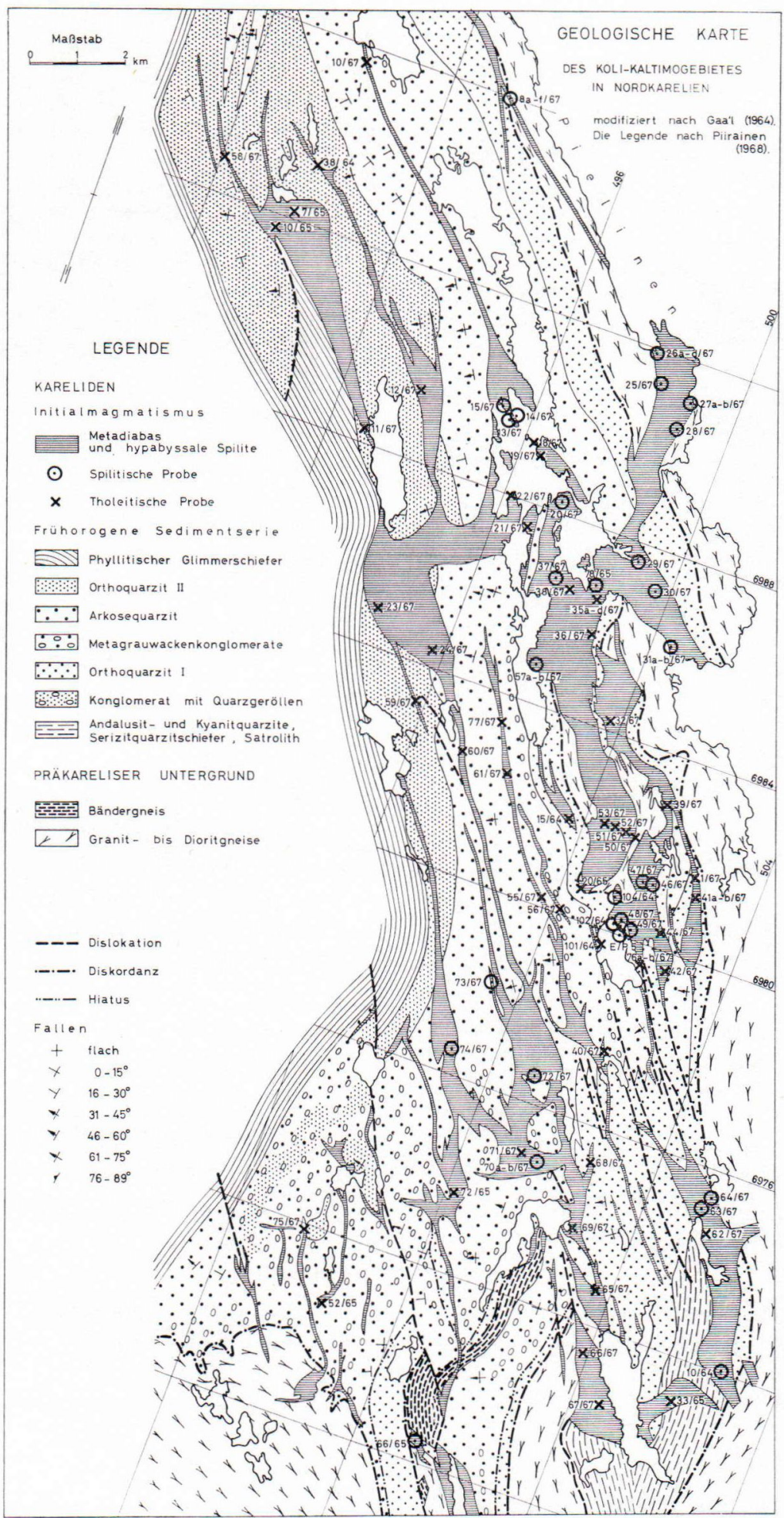


Sedimentserie oder auf dem Jatul weitverbreitet sind, und da sie von Graniten durchschnitten werden, hat Piirainen (1968) die polymikten Konglomerate, die Gaál für Bodenkonglomerate der karelischen Molasse hält, als eine intraformationale Bildung des Jatuls und die schneidenden Erstarrungsgesteine als Produkte des initialen Magmatismus gedeutet.

Für die Laboruntersuchung der Erstarrungsgesteine des Gebiets habe ich über hundert Proben gesammelt, von denen später mikroskopische, röntgendiffraktometrische, mikroanalysatorische und chemische Proben genommen worden sind. Die Lage der Proben, die bei der Untersuchung von Bedeutung waren, ist in der Abb. 1 verzeichnet. Nach den Untersuchungen kann man die Erstarrungsgesteine in zwei verschiedene Gruppen teilen, die sich nach ihrem Vorkommen, ihrer Struktur, chemischen Zusammensetzung und ihrem Mineralbestand sowie ihrer Entstehung nach voneinander unterscheiden. Die eine Gruppe wird hier als tholeitische, die andere als spilitische Assoziation bezeichnet.

\section{Tholeitische Assoziation}

Die Gesteine, die ihrer chemischen Zusammensetzung nach den Tholeiten entsprechen,

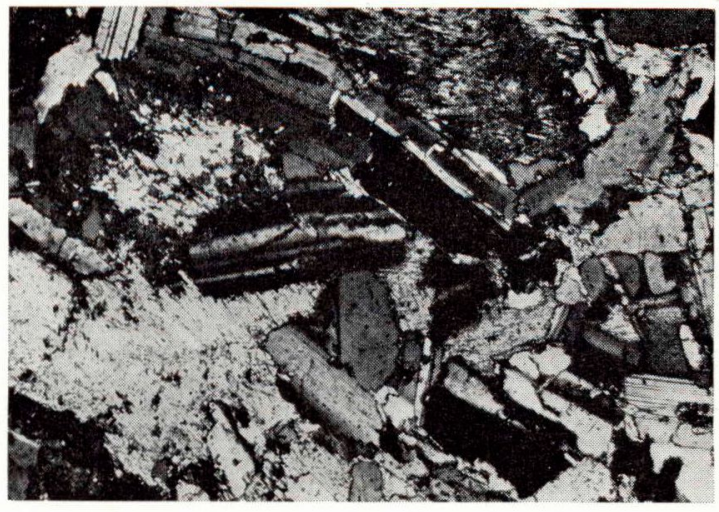

Abb. 2. Uralitdiabas. Probe 12/67. $x=493.55, y=$ 6989.55. Nic. +. Vergr. $44 \mathrm{mal}$.

bilden schmale schneidende Gänge. Ihre Farbe ist dunkelgrün, und sie haben eine feinkörnige Struktur, man kann aber in ihnen schon makroskopisch einzelne Mineralkörner unterscheiden. Die Gänge haben Bewegungen mitgemacht, wobei sie stellenweise krumm, und parallel zu den Gang schieferig geworden sind. Der Schieferungsgrad weckselt jedoch so $a b$, dass einige stark schieferig sind, anderen dagegen die Schieferung ganz und gar fehlen kann. In dem letzteren Fall ist die ophitische Struktur des ursprünglichen Diabases in dem Gestein zu sehen (Abb. 2).

TABelle 1.

Mineralzusammensetzung von sechs tholeitischen Proben und ihr durchschnittlicher Wert in Volumprozenten. Die Analysen sind mit einem Punktzähler gemacht worden.

\begin{tabular}{|c|c|c|c|c|c|c|c|}
\hline & 1. & 2. & 3. & 4. & 5. & 6. & 7. \\
\hline 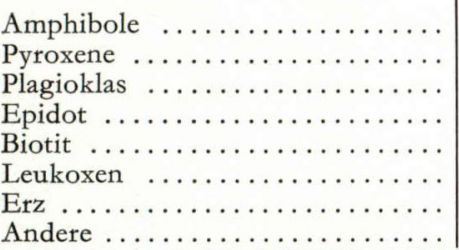 & $\begin{array}{r}32.3 \\
29.7 \\
34.7 \\
- \\
- \\
- \\
- \\
0.1\end{array}$ & $\begin{array}{r}49.1 \\
44.6 \\
2.0 \\
1.7 \\
- \\
1.4 \\
1.2\end{array}$ & $\begin{array}{r}51.9 \\
41.6 \\
4.8 \\
- \\
- \\
1.3 \\
0.4\end{array}$ & $\begin{array}{r}57.9 \\
-3 \\
31.0 \\
0.5 \\
0.9 \\
0.4 \\
\end{array}$ & $\begin{array}{r}68.2 \\
-\overline{15.9} \\
8.1 \\
5.4 \\
2.1 \\
- \\
0.3\end{array}$ & $\begin{array}{r}59.5 \\
- \\
9.3 \\
27.6 \\
1.4 \\
1.0 \\
1.1 \\
0.1\end{array}$ & $\begin{array}{r}58.1 \\
38.2 \\
1.5 \\
1.9 \\
0.4\end{array}$ \\
\hline & 100.0 & 100.0 & 100.0 & 100.0 & 100.0 & 100.0 & $1) 0.1$ \\
\hline
\end{tabular}

1. Probe Nr. $1 / 67, \mathrm{x}=503.10, \mathrm{y}=6980.50$, Diabas

2. Probe Nr. 22/67, $\mathrm{x}=496.15, \mathrm{y}=6988.00$, Uralitdiabas

3. Probe Nr. $12 / 67, \mathrm{x}=493.55, \mathrm{y}=6989.55$, Uralitdiabas

4. Probe Nr. 62/67, $\mathrm{x}=505.35, \mathrm{y}=6974.80$, Grünsteindiabas

5. Probe Nr. 71/67, $\mathrm{x}=501.30, \mathrm{y}=6975.00$, Grünsteindiabas

6. Probe Nr. $101 / 64, x=501.25, y=6979.70$, Grünsteindiabas

7. Durschnittliche Mineralzusammensetzung der obengenannten Proben. 
Tabelle 2 .

Chemische Analysen von tholeitischen Gesteinen des Koli-Kaltimogebiets.

\begin{tabular}{|c|c|c|c|}
\hline & $1 / 67$ & $12 / 67$ & $101 / 64$ \\
\hline 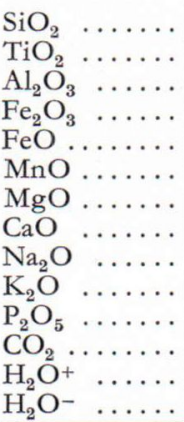 & $\begin{array}{r}49.61 \\
0.88 \\
13.80 \\
2.12 \\
8.81 \\
0.17 \\
8.70 \\
12.38 \\
1.71 \\
0.20 \\
0.04 \\
0.16 \\
0.77 \\
0.09\end{array}$ & $\begin{array}{r}49.58 \\
0.84 \\
17.23 \\
2.00 \\
7.34 \\
0.14 \\
6.35 \\
12.24 \\
1.92 \\
0.24 \\
0.03 \\
0.18 \\
1.33 \\
0.09\end{array}$ & $\begin{array}{r}50.25 \\
1.56 \\
13.19 \\
4.94 \\
9.05 \\
0.20 \\
6.32 \\
10.00 \\
1.76 \\
0.30 \\
0.20 \\
0.12 \\
1.96 \\
0.03\end{array}$ \\
\hline & 99.44 & 99.51 & 99.88 \\
\hline
\end{tabular}

$1 / 67$ Diabas; $x=503.10, y=6980.50$

$12 / 67$ Uralitdiabas; $x=493.55, y=6989.55$

$101 / 64$ Grünsteindiabas; $x=501.25, y=6979.70$

Der Mineralbestand und die chemische Zusammensetzung der Gesteine sind in den Tabellen 1 und 2 dargestellt. Die den chemischen Zusammensetzungen entsprechenden Normen und Niggliwerte sind in den Tabellen 3 und 4 zu sehen.

Die wenig umgewandelten Typen enthalten als Hauptgemengteil Plagioklas $\left(\mathrm{An}_{50-60}\right)$, Hypersthen $\left(2 \mathrm{~V}_{\mathrm{x}}=52^{\circ}, \mathrm{Z}-\mathrm{X}=0.013\right)$, Augit $(\mathrm{c} \wedge \mathrm{Z}$

Tabelle 3.

Normen von tholeitischen Gesteinen des Koli-Kaltimogebiets. Die entsprechenden Analysen sind in der Tabelle 2 dargestellt.

\begin{tabular}{l|r|r|r}
\hline & \multicolumn{1}{|c|}{$1 / 67$} & $12 / 67$ & $101 / 64$ \\
\hline $\mathrm{Q} \ldots \ldots \ldots \ldots$ & 0.6 & 2.1 & 8.3 \\
$\mathrm{Or} \ldots \ldots \ldots$ & 1.0 & 1.5 & 2.0 \\
$\mathrm{Ab} \ldots \ldots \ldots$ & 16.0 & 17.5 & 16.5 \\
$\mathrm{An} \ldots \ldots \ldots$ & 29.8 & 38.8 & 28.5 \\
$\mathrm{CaCO} \ldots \ldots$ & 0.4 & 0.4 & 0.4 \\
$\mathrm{Ap} \ldots \ldots \ldots$ & - & & 0.3 \\
$\mathrm{Di} \ldots \ldots \ldots$ & 25.2 & 18.0 & 17.6 \\
$\mathrm{Hy} \ldots \ldots \ldots$ & 23.4 & 18.2 & 18.8 \\
$\mathrm{Il} \ldots \ldots \ldots$ & 1.2 & 1.2 & 2.4 \\
$\mathrm{Mt} \ldots \ldots \ldots$ & 2.3 & 2.3 & 5.4
\end{tabular}

Tabelle 4.

Niggliwerte der tholeitischen Gesteine des Koli-Kaltimogebiets. Die entsprechenden Analysen sind in der Tabelle 2 dargestellt.

\begin{tabular}{l|c|c|c}
\hline & $1 / 67$ & $12 / 67$ & $101 / 64$ \\
\hline si $\ldots \ldots \ldots \ldots$ & 109.7 & 116.2 & 121.8 \\
al $\ldots \ldots \ldots \ldots$ & 17.9 & 23.8 & 18.2 \\
fm $\ldots \ldots \ldots$ & 48.8 & 40.6 & 50.7 \\
$\mathrm{e} \ldots \ldots \ldots \ldots$ & 29.3 & 30.8 & 26.0 \\
alk $\ldots \ldots \ldots$ & 4.0 & 4.8 & 4.5 \\
$\mathrm{k} \ldots \ldots \ldots \ldots$ & 0.07 & 0.09 & 0.10 \\
mg $\ldots \ldots \ldots$ & 0.59 & 0.55 & 0.45 \\
qz $\ldots \ldots \ldots$ & -6.3 & -3.2 & -3.8
\end{tabular}

$=36^{\circ}-42^{\circ}, 2 \mathrm{~V}_{\mathrm{z}}=46^{\circ}-48^{\circ}, \mathrm{Z}-\mathrm{X}=0.025$ ) und Hornblende ( $\mathrm{Z}=$ blaugrün-olivingrün, $\mathrm{Y}=$ hellgrün, $\mathrm{X}=$ farblos; $\mathrm{c} \wedge \mathrm{Z}=20^{\circ}-23^{\circ}$; $2 \mathrm{~V}_{\times}=70^{\circ}-72^{\circ}$ und $\left.80^{\circ} ; \mathrm{Z}-\mathrm{X}=0.020-0.023\right)$.

Der Hypersthen und der Augit sind jedoch unbeständig geworden: Der Hypersthen hat sich in Tremolit und der Augit in Hornblende umgewandelt. Die wenig umgewandelten Typen sind danach zu schliessen Uralitdiabase. Als Ủbergemengteile enthalten sie Titanomagnetit, Titanit, Magnetkies und Kupferkies. Es ist bemerkenswert, dass der Titanomagnetit in Gestalt allotriomorpher Körner vorkommt (Abb. 4). Er vertritt also die letzte Kristallisation in dem Gesteine.

Oft ist die Umwandlung der Diabase noch nicht bei dem Uralitdiabas stehen geblieben. Der anorthitreiche Plagioklas ist umbeständig geworden und in Epidot, Albit und Quarz zerfallen. In den Gesteinen kommen keine Pyroxene vor, und auch die Hornblende hat sich teilweise rekristallisiert. Sie bildet jetzt olivingrüne zerrissene Körner und blaugrüne splitterige Kristalle. Hierbei ist die ursprüngliche Struktur beinahe völlig verschwunden (Abb. 3). Dieses Gestein ist Grünsteindiabas genannt worden. Neben der Hornblende, dem Epidot, Quarz und Albit enthält es ein wenig Karbonat, Titanit, Titanomagnetit, Schwefelkies und Kupferkies. Die Magnetitlamellen des Titanomagnetits werden von Silikaten wie Hornblende, Biotit und Chlorit verdrängt (Abb. 5). 


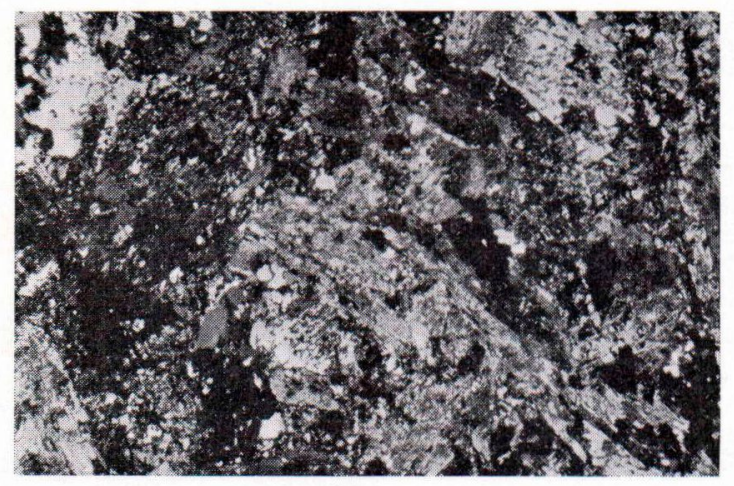

Abb. 3. Grünsteindiabas. Probe 101/64. $\mathrm{x}=501.25$, y $=6979.70$. Nic. + . Vergr. 32mal.

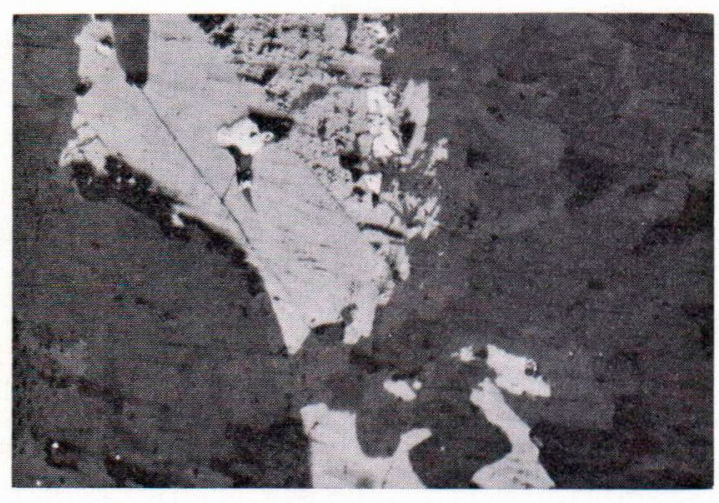

Abb. 4. Allotriomorpher Titanomagnetit in Uralitdiabas. Probe $1 / 67 . \quad x=503.10, y=6980.50$. Vergr. 60mal.

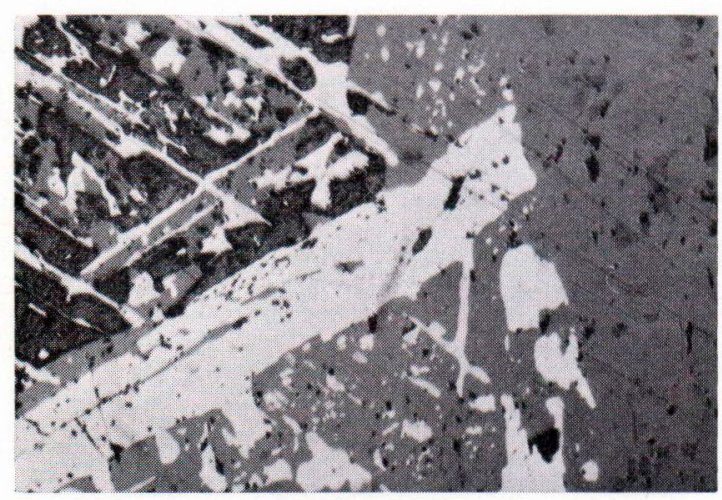

Abb. 5. Magnetitkomponente des Titanomagnetits in Grünsteindiabasen wird von Silikaten verdrängt, so dass ein Ilmenitskelett zurückgeblieben ist. Probe 35/67. $\mathrm{x}=498.65, \mathrm{y}=6986.70$. Vergr. $150 \mathrm{mal}$.
An einigen Stellen ist die Umwandlung noch fortgeschtritten. Die Hornblende ist unbeständig geworden, und sie ist in Chlorit und in Epidot zerfallen. Die Gesteine, die diese Umwandlung durchgangen sind, sind oft sehr schieferig. Mit Rücksicht auf den Mineralbestand und die Struktur können sie als Grünschiefer bezeichnet werden. Die Grünschiefer haben jedoch keine, weite Verbreitung.

Von den Metadiabasen sind drei chemische Analysen gemacht worden (s. Tab. 2), die verschiedengradig metamorphosierte Typen repräsentieren. Eine Analyse vertritt die Uralitdiabase, die noch Pyroxene enthalten, und die anderen vertreten Typen, bei denen die Umwandlung viel weiter fortgeschtritten ist. Die Analysen gleichen sich untereinander und dem tholeitischen Magmatyp sehr (Kennedy 1933, s. 241). Weshalb man annehmen kann, dass es sich wirklich um verschiedengradig metamorphosierte Diabase handelt.

\section{Spilitische Assoziation}

Die Gesteine der spilitischen Assoziation kommen in Form dicker schichtenparalleler Platten vor. Wie es auf der Karte (Abb. 1) zu sehen ist, bilden sie stellenweise sogar $2 \mathrm{~km}$ breite Zonen. Mit Rücksicht auf das seichte Fallen und die treppenförmige Struktur des Felsgrunds lässt sich annehmen, dass die Dicke der Platten nur höchstens $0.5 \mathrm{~km}$ beträgt. Wenn man von den östlichen Rändern der Gänge zu den westlichen übergeht, also von unten nach oben, kann man Veränderungen in der Korngrösse, der Struktur, dem Mineralbestand und der chemischen Zusammensetzung feststellen. Die Körner sind kleiner als $1 \mathrm{~mm}$. Nach Westen wird das Gestein heller, stellenweise rötlich. Dabei wächst die Korngrösse. Bei den gröbsten Typen kann der Durchmesser beinahe $10 \mathrm{~mm}$ sein. Die Gänge haben Bewegungen mitgemacht, da die Schieferung an vielen Stellen, besonders in den äusseren Teilen der Gänge, vorkommt. Im allgemeinen bleibt die Schieferung jedoch gering. 
TABELle 5 .

Mineralzusammensetzung der Gesteine der spilitischen Assoziation im Koli-Kaltimogebiet. Die analysen sind mit einem Punktzähler gemacht worden.

\begin{tabular}{|c|c|c|c|c|c|c|c|c|c|}
\hline & 1. & 2. & 3. & 4. & 5. & 6. & 7. & 8. & 9. \\
\hline$\ldots \ldots \ldots \ldots$ & 53.2 & 14.1 & - & - & - & 一 & - & - & - \\
\hline Amphibole ............ & 34.0 & 56.3 & 57.2 & 63.5 & 53.8 & 474 & 51.8 & 59.8 & - \\
\hline Biotit $\ldots \ldots \ldots \ldots \ldots \ldots$ & 一 & 一 & - & 一 & - & - & - & 2.4 & 17.3 \\
\hline Titanit und Leukoxen .... & 1.4 & 2.0 & 0.8 & 一 & 1.9 & 2.9 & 0.9 & 2.7 & - \\
\hline Titanomagnetit $\ldots \ldots \ldots \ldots$ & - & 2.4 & - & 3.5 & 3.3 & 6.6 & 0.5 & 1.1 & 5.2 \\
\hline Chlorit ............ & 5.1 & - & - & 2.8 & - & - & 6.4 & - & 6.8 \\
\hline Plagioklas ............ & 6.1 & 22.0 & 24.6 & 26.7 & 30.0 & 30.6 & 30.7 & 33.8 & 35.4 \\
\hline Epidot $\ldots \ldots \ldots \ldots \ldots$ & 一 & 2.9 & 15.6 & 1.4 & 9.8 & 12.1 & 1.3 & 一 & - \\
\hline Karbonat ............ & 一 & - & 1.6 & - & 一 & 一 & 7.8 & - & 28.6 \\
\hline Quarz $\ldots \ldots \ldots \ldots \ldots \ldots$ & - & 一 & - & 1.8 & - & - & 0.6 & 一 & 6.4 \\
\hline Andere $\ldots \ldots \ldots \ldots \ldots$ & 0.2 & 0.3 & 0.2 & 0.3 & 1.2 & 0.4 & - & 0.2 & 0.3 \\
\hline
\end{tabular}

\begin{tabular}{|c|c|c|c|c|c|c|c|c|c|c|}
\hline & 10. & 11. & 12. & 13. & 14. & 15. & 16. & 17. & 18. & 19. \\
\hline Pyroxene $\ldots \ldots \ldots \ldots \ldots$ & - & - & - & - & - & - & - & - & 一 & - \\
\hline Amphibole...$\ldots \ldots \ldots$. & 42.7 & 43.2 & 32.1 & 21.2 & 33.5 & 23.5 & 13.8 & - & 17.8 & 21.5 \\
\hline Biotit $\ldots \ldots \ldots \ldots \ldots \ldots$ & 4.0 & 4.0 & 4.5 & 2.2 & 1.8 & 4.8 & 9.6 & 4.4 & 6.7 & 6.7 \\
\hline Titanit und Leukoxen .... & 1.1 & - & - & 2.3 & - & - & - & - & 2.6 & - \\
\hline Titanomagnetit $\ldots \ldots \ldots$. & 8.0 & 1.7 & 5.2 & 10.1 & 10.6 & 7.9 & 10.9 & 3.9 & 7.6 & 4.9 \\
\hline Chlorit $\ldots \ldots \ldots \ldots \ldots$ & 8.3 & - & - & 9.8 & - & 1.0 & 2.6 & 16.4 & - & 1.8 \\
\hline Plagioklas $\ldots \ldots \ldots \ldots \ldots$. & 35.5 & 39.7 & 42.1 & 48.8 & 50.6 & 53.7 & 56.3 & 59.1 . & 62.5 & 62.7 \\
\hline Epidot $\ldots \ldots \ldots \ldots \ldots$ & - & 6.7 & 15.8 & 1.0 & 2.9 & 1.3 & 5.2 & - & 2.7 & 1.5 \\
\hline Karbonat $\ldots \ldots \ldots \ldots \ldots$ & - & 3.2 & - & 2.0 & - & - & - & - & - & - \\
\hline Quarz $\ldots \ldots \ldots \ldots \ldots \ldots$ & - & - & - & 2.3 & - & 6.8 & 1.5 & 15.0 & - & - \\
\hline Andere $\ldots \ldots \ldots \ldots \ldots$ & 0.4 & 0.5 & 0.3 & 0.3 & 0.7 & 1.0 & 0.1 & 1.2 & 0.1 & 0.9 \\
\hline
\end{tabular}

1. Probe Nr. $27 \mathrm{a} / 67, \mathrm{x}=498.95, \mathrm{y}=6991.25$ )

2. Probe Nr. 28/67, $\mathrm{x}=498.95, \mathrm{y}=6990.60$ Peridotite

3. Probe Nr. 3/67, $\mathrm{x}=491.90, \mathrm{y}=6999.70$

4. Probe Nr. 47/67, $x=501.65, y=6981.30$ '

5. Probe Nr. 30/67, $x=499.70, y=6987.20$

6. Probe Nr. $31 \mathrm{~b} / 67, \mathrm{x}=500.40, \mathrm{y}=6986.20$

7. Probe Nr. $8 \mathrm{a} / 67, \mathrm{x}=493.15, \mathrm{y}=6996.00$

8. Probe Nr. $48 / 67, x=501.40, y=6980.25$

9. Probe Nr. $14 / 67, x=495.65, y=6989.55$ hornblendereiche Albitdiabase

10. Probe Nr. $64 / 67, x=505.25, y=6974.50$

11. Probe Nr. $57 \mathrm{a} / 67, \mathrm{x}=497.80, \mathrm{y}=6984.70$

12. Probe Nr. 25/67, $x=498.30, y=6991.35$

13. Probe Nr. $9 a / 67, x=490.65, y=6997.55$

14. Probe Nr. $13 / 67, x=495.60, y=6989.50$

15. Probe Nr. 29/67, $x=499.10, y=6987.75$

16. Probe Nr. $72 / 67, x=500.95, y=6976.55$

17. Probe Nr. $9 \mathrm{~d} / 67, \mathrm{x}=490.65, \mathrm{y}=6997.55$ albitreiche Albitdiabase

18. Probe Nr. $10 / 64, x=506.80, y=6972.10$

19. Probe Nr. $37 / 67, x=496.70, y=6986.75$ )

Die mikroskopische Untersuchung hat gezeigt, dass der Amphibol und der Plagioklas bei allen Typen als Hauptmineralien auftreten. Als Nebenund Übergemengteile können Pyroxen, Epidot, Biotit, Cholorit, Karbonat, Quarz, Titanit, Erzmineralien und Apatit vorkommen. Die Proportionen der Mineralien wechseln ständig (s.
Tab. 5). In den dunklen Gesteinen kann die Menge der dunklen Mineralien $90 \%$ und in den hellen Typen die der hellen Mineralien $65 \%$ betragen.

Der Amphibol kommt in den dunklen Gesteinen in Form eigenförmiger Kristalle und als Pseudomorphose nach Pyroxen (s. Abb. 6 und 


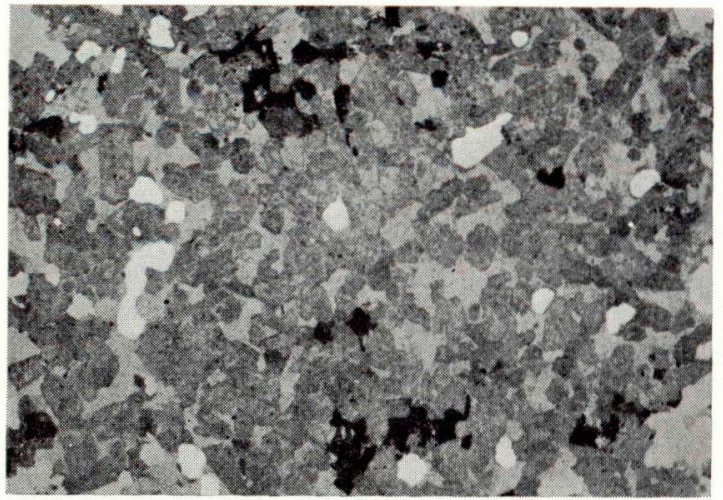

Abb. 6. Peridotit mit hypidiomorpher Struktur, wo der Albit (hell) in Form von allotriomorphen Körnern zwischen idiomorphen Pyroxenkristallen (grau) liegt. Der Pyroxen hat sich teilweise in Hornblende umgewandelt. Probe 28/68. $\mathrm{x}=498.95, \mathrm{y}=6990.60$. Nic. $/ /$. Vergr. $8 \mathrm{mal}$.

7), in den hellen Gesteinen in Form langer spilitteriger Kristalle vor, die zwischen den Plagioklaskristallen liegen (s. Abb. 8 und 9). Die optischen Eigenschaften des Minerals variieren sehr, sogar in demselben Kristall. 44 Bestimmungen weisen folgende Varianten auf:

$$
\begin{aligned}
\mathrm{X} & =\text { farblos-hellgelb } \\
\mathrm{Y} & =\text { farblos-hellgrün-grün } \\
\mathrm{Z} & =\text { hellgrün-blaugrün } \\
2 \mathrm{~V} \times & =60^{\circ}-64^{\circ}, 68^{\circ}-72^{\circ}, 78^{\circ}-80^{\circ} \\
\mathrm{c} \wedge \mathrm{Z} & =12^{\circ}-24^{\circ} \\
\mathrm{Z}-\mathrm{X} & =0.014-0.024
\end{aligned}
$$

Auf Grund der optischen Eigenschaften des Amphibols kann man feststellen, dass es sich in den meisten Fällen um Hornblende handelt. Manchmal hat der Amphibol einen kleinen $c \wedge Z$, einen grossen $2 V_{x}$, eine niedrige Interferenzfarbe und einen schwachen Pleochroismus was darauf hindeutet, dass es sich dabei um Tremolit handelt. Als Umwandlungsprodukt enthält der Amphibol Biotit und Chlorit.

Der Pyroxen wird nur bei den dunklen Typen angetroffen. Er kommt in Form eigenförmiger Kristalle vor, die sich teilweise in blaugrüne Hornblende umgewandelt haben. Die optischen Eigenschaften des Minerals schwanken einiger-

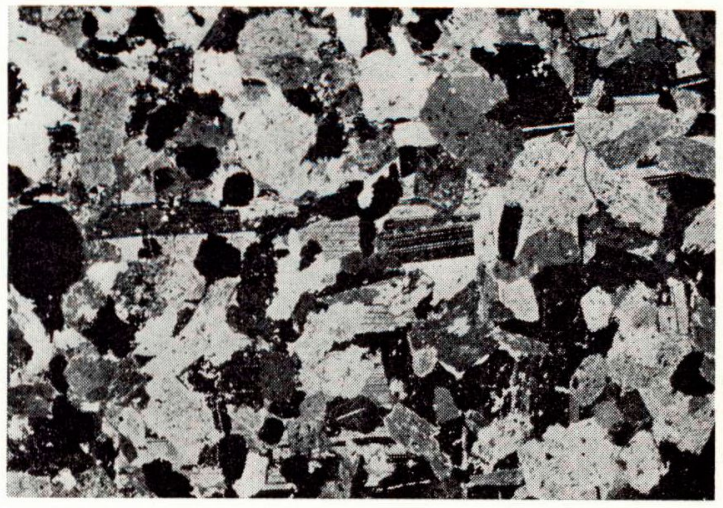

Abb. 7. Hornblendereicher Albitdiabas mit hypidiomorpher Struktur, wo der Albit Hornblendekristalle in sich einschliesst. Die schwarzen Flecken sind Spuren der beim Schleifen lose gewordenen Hornblendekristalle. Probe 53/64. $\mathrm{x}=490.75, \mathrm{y}=7001.80$. Nic. + . Vergr. $6 \mathrm{mal}$.

massen: $c \wedge Z$ variiert $38^{\circ}-46^{\circ}$. Die Interferenzfarbe berträgt 0.025 .

Der Plagioklas kommt in den dunklen Gesteinen in Gestalt grosser Kristalle vor, die Pyroxen- und Hornblendekristalle in sich einschliessen (s. Abb. 6 und 7). In den hellen Typen erscheint er dagegen in Form von tafeligen Kristallen, die oft, dem Albitgesetz gemäss eine Zwillingsbildung haben (s. Abb. 8 und 9). Aus den optischen Eigenschaften kann man schliessen, dass der Plagioklas Albit ist. Sein Anorthitgehalt schwankt zwischen $2-7 \%$. In dem Albit kommen Epidot und auch blaugrüne Hornblende als supmikroskopisch kleine Kristalle vor.

Der Epidot bildet ausser den supmikroskopisch kleinen Körnern im Albit auch selbständige kleine Kristalle. Die optischen Eigenschaften zeigen, dass der Epidot pistasitreich ist.

Der Biotit ist nur in den hellen Typen anzutreffen. Er erscheint in Form kleiner Schuppenanhäufungen zwischen den Albitkristallen. Dazu tritt er auch als Umwandlungsprodukt nach dem Amphibol auf. Selbst hat das Mineral sich teilweise in Chlorit umgewandelt. Der Chlorit kommt nur als Umwandlungsprodukt nach dem Biotit, dem Amphibol und dem Pyroxen vor. 


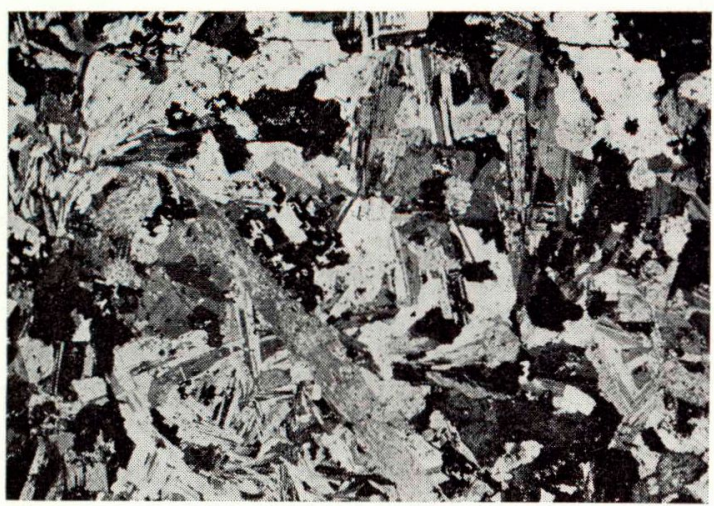

Abb. 8. Albitreicher Albitdiabas mit hypidiomorpher Struktur, wo der Albit in Form von idiomorphen Kristallen und die Hornblende in Form von langen splitterigen Körnern vorkommen. Probe 102/64. x= 501.35, y $=6980.20$. Vergr. 5.5mal.

Einige Typen enthalten sehr viel Karbonat. Es kommt in Form unbestimmter Körner zwischen den anderen Mineralien vor, die es auch oft verdrängt. Nach den Brechungskoeffizienten zu schliessen handelt es sich um Kalzit.

Für die Untersuchung der Erzmineralien in den spilitischen Gesteinen sind 17 Anschliffe gemacht worden. In diesen Anschliffen sind folgende Erzmiralien gefunden worden: Titanomagnetit, Magnetit, Ilmetit, Hämatit, Schwefelkies, Magnetkies, Kupferkies, Bornit, Kupferglanz und Markasit.

Von den oxydischen Erzmineralien ist Titanomagnetit am reichlichsten vorhanden. In den dunklen Typen fehlt er beinahe völlig, während er in den hellen Gesteinen bis zu 10 \% vorhanden sein kann. Der Titanomagnetit bildet idiomorphe Kristalle (s. Abb. 10). Nach seiner Entstehung hat er jedoch einige Umwandlungsprozesse durchgemacht. Die Magnetitkomponente ist teilweise in Hämatit oxydiert, und die Ilmenitkomponente in Leukoxen umgewandelt (s. Abb. 11). Nach röntgenographischen Untersuchungen besteht der Leukoxen aus Titanit.

Von den sulfidischen Erzmineralien sind Schwefelkies und Kupferkies am reichlichsten

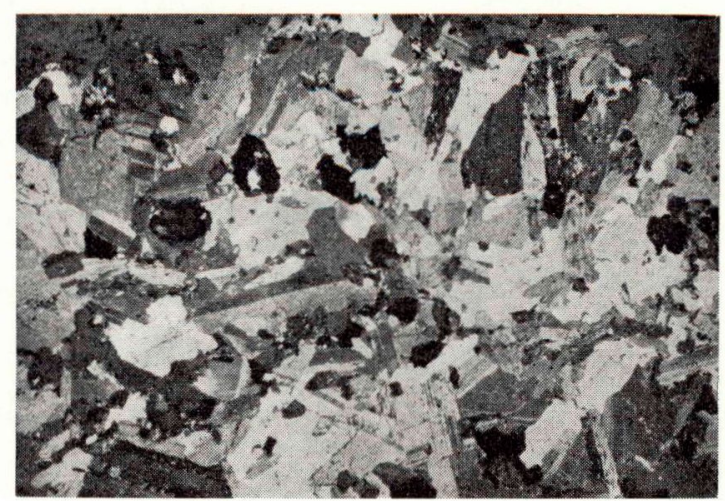

Abb. 9. Albitreicher Albitdiabas mit hypidiomorpher Struktur, wo die Hornblende (grau) in geringer Masse zwischen idiomorphen Albit- und Titanomagnetitkristallen (hell und scwarz) vorkommt. Probe 78/65. $\mathrm{x}=$ 498.45, y $=6986.90$. Vergr. 6mal.

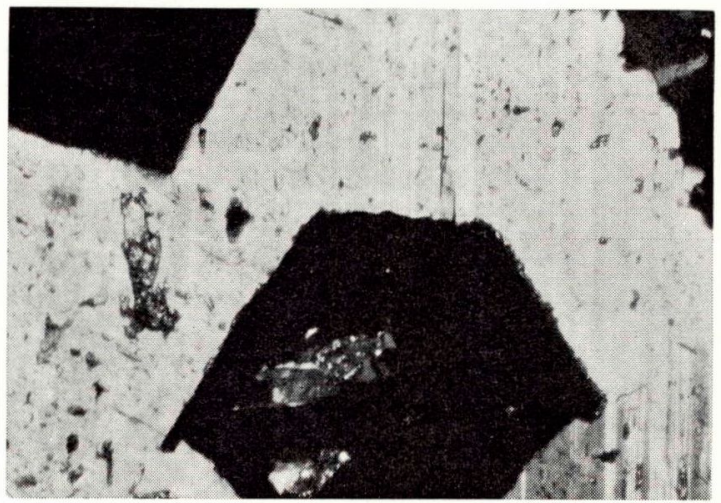

Abb. 10. Idiomorpher Titanomagnetit in albitreichem Albitdiabas. Probe 78/65. $x=498.45, \quad y=6986.90$. Nic. +. Vergr. $32 \mathrm{mal}$.

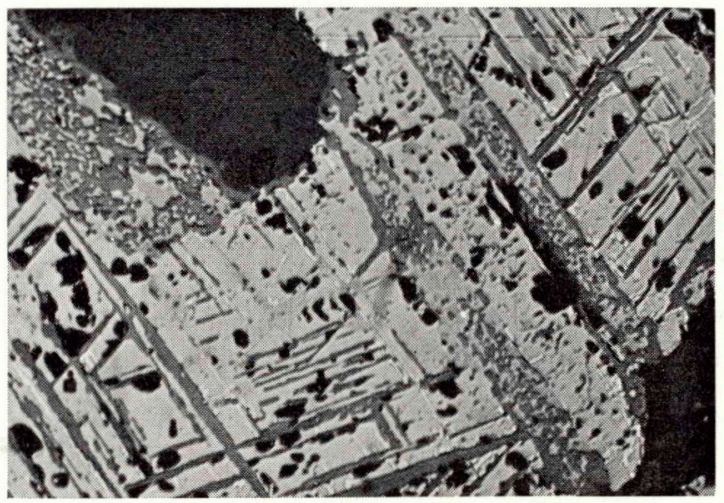

Abb. 11. Ilmenitlamellen des Titanomagnetits sind von Titanit verdrängt und dessen Magnetitkomponente hat sich teilweise in Hämatit umgewandelt. Dieselbe Probe wie oben. Vergr. 150mal. 


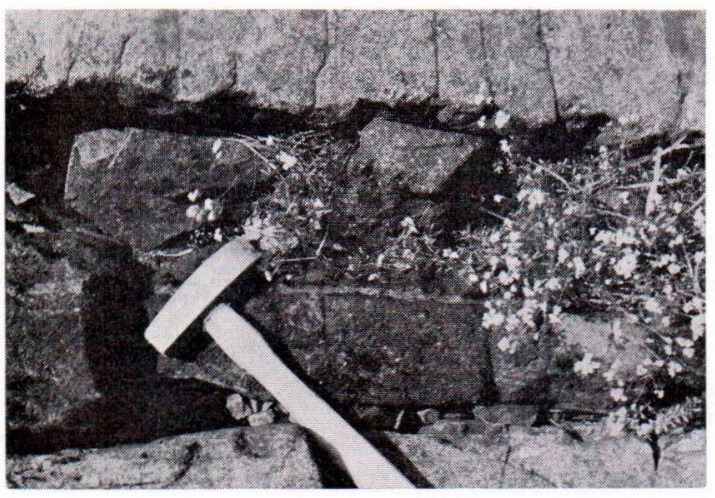

Abb. 12. Albit-Ankeritgang in hornblendereichem Albitdiabas. $\mathrm{x}=491.90, \mathrm{y}=6999.70$.

zu finden. Der Schwefelkies bildet oft eigenförmige Kristalle. Der Kupferkies, sowie die anderen Sulfide treten dagegen in Form unbestimmter Körner auf. Der Bornit hat sich oft in Kupferglanz und der Magnetkies in Markasit umgewandelt. Am reichlichsten kommen die Sulfide in Gesteinsspalten vor.

Die Gesteine der spilitischen Assoziation des Koli-Kaltimogebiets können zweierlei Struktur aufweisen. In dem einen Typ, der in den dunklen Gesteinen auftritt, kommen der Pyroxen und der Amphibol als idiomophe Kristalle vor, die der Albit in sich einschliesst. In dem anderen Typ, der in den hellen Gesteinen angetroffen wird, bildet der Albit idiomorphe Kristalle, zwischen denen Hornblende zu sehen ist. Die beiden Type sind hypidiomorph, und zwar so, dass in den dunklen Gesteinen zuerst der Pyroxen und der Amphibol und darauf der Albit sich kristallisiert haben (s. Abb. 6 und 7), während die Kristallisationsfolge in den hellen Gesteinen umgekehrt ist: zuerst hat sich der Albit und dann die Hornblende kristallisiert (s. Abb. 8 und 9).

Die Gesteine der spilitischen Assoziation werden von verschiedenen Mineralgängen durchschnitten. Mit Rücksicht auf ihre Mineralzusammensetzung kann man in ihnen folgende Gänge unterscheiden: Albitgänge, Albit-Ankeritgänge und kieshaltige Quarz-Karbonatgänge.
Ausser die Intrusive selbst können diese Gänge auch in der Nähe der Intrusive Metadiabase sowie Metapsephite und Metapsammite durchschneiden.

Ein Albitgang wird in Paukkajanvaara (Probe 30T, s. Abb. 1) angetroffen. Neben den Albit $\left(\mathrm{An}_{5}\right)$ kommt als Hauptgemengteil auch Muskovit $\left(\mathrm{n}_{\mathrm{z}}=1.601,2 \mathrm{~V}_{\mathrm{x}}=\mathrm{n} .45^{\circ}\right)$ vor. Als Neben- und Übergemengteil enthält der Gang dazu Quarz, Kalsit, Turmalin, Chlorit und Rutil. Der Albit kann stellenweise in so grossen Kristallen auftreten, dass das Aussehen des Gesteins auf Pegmatit hindeutet. Der Gang ist $1-5 \mathrm{~m}$ breit, und er kommt als Lagergang weit in der Bodenformation vor. In der Grube von Paukkajanvaara ist zu sehen, dass der Gang den Metadiabas schneidet.

Die Albit-Ankeritgänge (Abb. 12) haben sich in einigen Intrusionen, wo sie ein Stockwerk bilden können, sehr weit verbreitet. Die Dicke

\section{TABELle 6.}

Chemische Analysen von spilitischen Gesteinen des Koli-Kaltimogebiets.

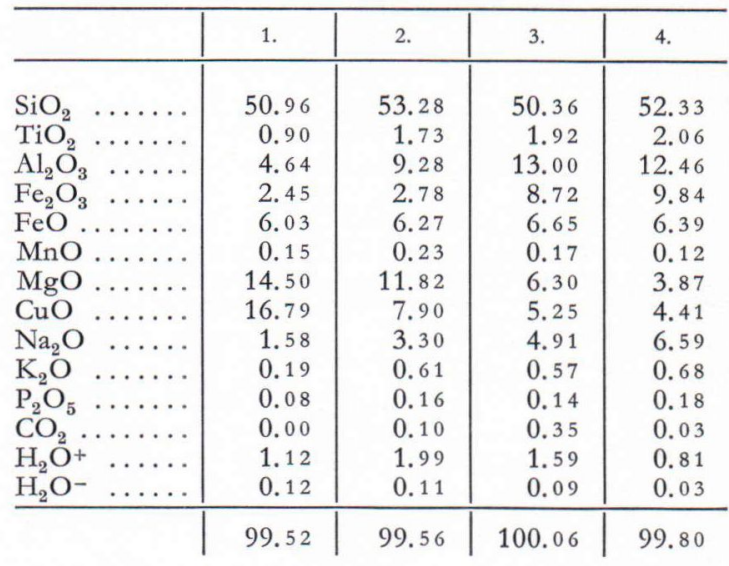

1. Probe 27a/67; Peridotit; $x=498.95, y=6991.25$. Anal. von R. Saikkonen. Geol, tutkimuslaitos 2. Probe 48/67; hornblendereicher Albitdiabas; $x=$ 501.40, y $=6980.25$. Anal. von R. Saikkonen. Geol. tutkimuslaitos

3. Probe 13/67; Albitdiabas; $x=495.60, y=6989.50$. Anal. von R. Saikkonen, Geol. tutkimuslaitos

4. Probe 10/64; albitreicher Albitdiabas; $x=506.80$, $y=6972.10$. Anal. von P. Ojanperä, Geol. tutkimuslaitos (s. Piirainen 1968) 
TABelle 7.

Normen von spilitischen Gesteinen des Koli-Kaltimogebiets. Die entsprechenden Analysen sind in der Tabelle 6 dargestellt.

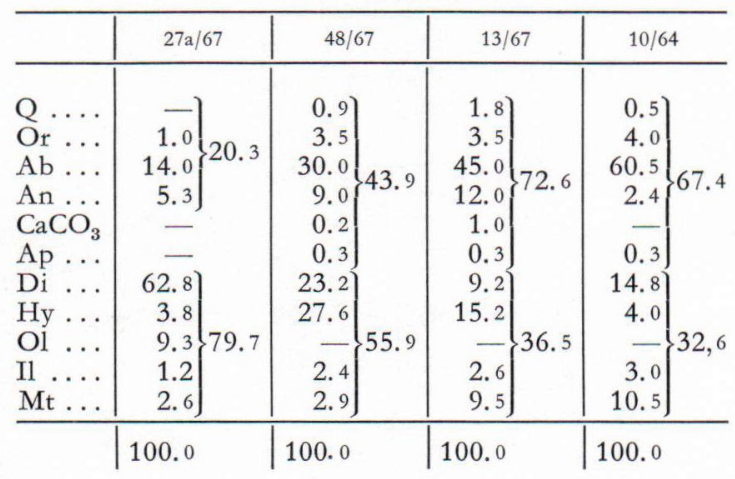

eines einzelnen Gangs in dem Stockwerk kann $0.5 \mathrm{~m}$, die Länge einige zehn Meter betragen. Neben dem Albit und Ankerit können diese Gänge als Neben- und U̇bergemengteil Tremolit, Chlorit, Kalzit, Quarz, Apatit, Muskovit und Erzmineralien enthalten. Die Menge der Erzmineralien ist gewöhnlich gering. In den äusseren Teilen der Gänge werden oft Titanomagmetitkörner angetroffen, deren Ränder korrodiert sind. In Spalten und auch zwischen anderen Mineralien kommen kleine Körner Schwefel-, Magnet- und Kupferkies in unbestimmter Form vor. Albit-Ankeritgänge können auch Metadiabase schneiden.

Die kieshaltigen Quarz-Karbonatgänge sind von alters her bekannt. Die bekanntesten von

TABelle 8.

Niggliwerte der spilitischen Gesteine des Koli-Kaltimogebiets. Die entsprechenden Analysen sind in der Tabelle 6 dargestellt.

\begin{tabular}{l|r|r|r|r}
\hline & $27 \mathrm{a} / 67$ & $48 / 67$ & $13 / 67$ & $10 / 64$ \\
\hline $\mathrm{si} \ldots .$. & 99.2 & 124.9 & 125.6 & 139.5 \\
$\mathrm{al} \ldots$. & 5.4 & 12.9 & 19.0 & 19.5 \\
$\mathrm{fm} \ldots$ & 56.4 & 59.1 & 54.2 & 49.8 \\
$\mathrm{e} \ldots .$. & 35.0 & 19.8 & 14.1 & 12.6 \\
alk $\ldots$ & 3.2 & 8.3 & 12.7 & 18.1 \\
$\mathrm{k} \ldots .$. & 0.07 & 0.10 & 0.07 & 0.06 \\
mg .. & 0.75 & 0.70 & 0.44 & 0.31 \\
qc ... & -13.6 & -8.3 & -25.2 & -32.9
\end{tabular}

ihnen sind die kleinen Erzlagerstätten von Kyykkä und Hokka (s. Abb. $152 / 65$ und 75/67). Zuletzt sind sie von Saksela (1960) beschrieben worden. Nach ihm können die Gänge folgende Erzmineralien enthalten: Kupferkies, Bornit, Kupferglanz, Schwefelkies, Magnetkies, Zinkblende, Bleiglanz, Magnetit, Ilmetit, Cubanit, Vallerit und Cobaltpentlandit. Die Gangart besteht hauptsächlich aus Quarz. Dazu kommen Kalzit, Dolomit, Chlorit, Hornblende und Titanit vor.

Von den Haupttypen der spilitischen Gesteinsassoziation sind chemische Analysen gemacht worden. Diese Analysen sind in der Tabelle $6 \mathrm{zu}$ sehen. Von den Analysen hat man auch Normen und Niggliwerte errechnet, die in den Tabellen 7 und 8 dargestellt sind. Die Analysen zeigen, dass am Übergang von den dunklen Typen $\mathrm{zu}$ den hellen die Menge des $\mathrm{Al}_{2} \mathrm{O}_{3}$, $\mathrm{Fe}_{2} \mathrm{O}_{3}, \mathrm{Na}_{2} \mathrm{O}$ und $\mathrm{K}_{2} \mathrm{O}$ wächst, während die Menge des $\mathrm{MgO}$ und $\mathrm{CaO}$ abnimmt. Dagegen bleibt die Menge des $\mathrm{SiO}_{2}$ und $\mathrm{FeO}$ in allen Typen ungefähr gleich.

\section{Die magmatische Entwicklung}

Beim Sinken des Bodens entstanden Spannungen in der Erdkruste, deren Nachlassen basisches Magma aus der Tiefe aufgetrieben hat. Die der chemischen Zusammensetzung entsprechenden Vulkanite sind in der karelischen Schieferzone auf dem Jatul weit verbreitet, und werden von Graniten durchschnitten. Daher ist anzunehmen, dass es sich um Produkte des initialen Magmatismus der karelisch-svekofennischen Orogenese handelt, und dass die basischen Gänge des Koli-Kaltimogebiets als Ausstiegskanäle dieses Magmatismus gedient haben.

Wie es in anderen Gebieten in bezug auf den initialen Magmatismus der Fall ist, hat er auch im Koli-Kaltimogebiet einerseits tholeitische und andererseits spilitische Gesteine erzeugt. Darüber, wie die spilitischen Gesteine sich zu den tholeitischen verhalten, hat man viel geschrieben. Einige sind der Ansicht, dass die Spilite 
durch eine $\mathrm{Na}$-Metasomatose von Basalten entstanden sind, andere halten sie für primär magmatisch. Schon früher habe ich geschrieben, dass man alle diese Erscheinungen, die in der spilitischen Gesteinen im Koli-Kaltimogebiet auftreten, mit Hilfe der Theorie "vom reichlich leichtflüchtige Bestandteile enthaltenden tholeitischen Stamm-Magma als Mutter der Spilite» deuten kann. Die initialen Magmen haben sich durch auf dem Meeresboden abgelagerte und vom Meereswasser gesättigte Sedimente intrudiert. Es ist denkbar, dass sie dabei von Wasser, Kohlendioxyd, Chlor und anderen leichtflüchtigen Bestandteilen kontaminiert worden sind. Jedenfalls ist das Magma im Koli-Kaltimogebiet reich an Kohlendioxyd und Wasser gewesen, was karbonathaltige Gänge und das weite Auftreten der wasserhaltigen Mineralien beweisen. Hier will ich jedoch nicht näher darauf eingehen, ob das Wasser und das Kohlendioxyd, auf die die Entstehung der spilitischen Gesteinsassoziation aus dem tholeitischen Stamm-Magma zurückzuführen ist, aus der Tiefe oder aus der evolutionärtransgressiven Sedimentserie stammen. Am Anfang der Intrusionen sind jedoch zuerst Diabase und darauf die spilitische Gesteinsassoziation entstanden, auf welche Altersfolge die Metadiabase durchschneidenden Albitpegmatitgänge hinweisen. Mit Rücksicht auf die kalte Umgebung und auf die daraus folgende schnelle Kristallisation ist diese Altersfolge zu verstehen. Während die Intrusionen sich fortgesetzt haben, haben sich die Randteile der schichtparallelen Intrusionen und die schmalen schneidenden Apophyse schnell auskristallisiert, die Kristallisation des mittleren Teils ist aber so langsam geschehen, dass eine Fraktionierung stattgefunden hat. So sind Peridotite, hornblendereiche Albitdiabase, in denen die Kristallisationsfolge Pyroxen-Hornblende-Albit $\mathrm{zu}$ sehen ist, und albitreiche Albitdiabase, in denen die Kristallisationsfolge Albit-Hornblende vorkommt, sowie pegmatitische, pneumatolytische und hydrotermische Gänge entstanden. Die bei der Kristallisation der weiten Intrusionen abgetrenn- ten verdunstbaren Bestandteile haben $\mathrm{Um}$ kristallisation in den schon kristallisierten Teilen hervorgerufen. Aus den Diabasen sind Uralitdiabase und aus ihnen weiter Grünsteindiabase und sogar Grünschiefer entstanden. Aber vor allem die früh kristallisierten Randteile der Lagergänge haben sich teilweise so umkristallisiert, dass die Hornblende in Form von langen splitterigen Kristallen vorkommt.

\section{Verteilung der schweren Metalle unter die Gesteine und ihre Mineralien}

Allgemeine im Bereich des initialen Magmatismus erzbildende Elemente sind $\mathrm{Cr}, \mathrm{Fe}, \mathrm{Ti}$, $\mathrm{Mn}$, Ni, Co und $\mathrm{Cu}$, weshalb gerade sie in dieser Arbeit betrachtet werden sollen. Es wird erläutert, wie diese Elemente sich unter die früher dargestellten Gesteinsarten und unter ihre Mineralien verteilt haben. Die Analysendaten sind in den Tabellen 9, 10 und 11 aufgeführt. Wie es in den Tabellen zu sehen ist, vertreten die analysierten Proben sowohl verschiedengradig metamorphosierte Diabase, Uralitdiabase und Grünsteindiabase als auch die Gesteine der

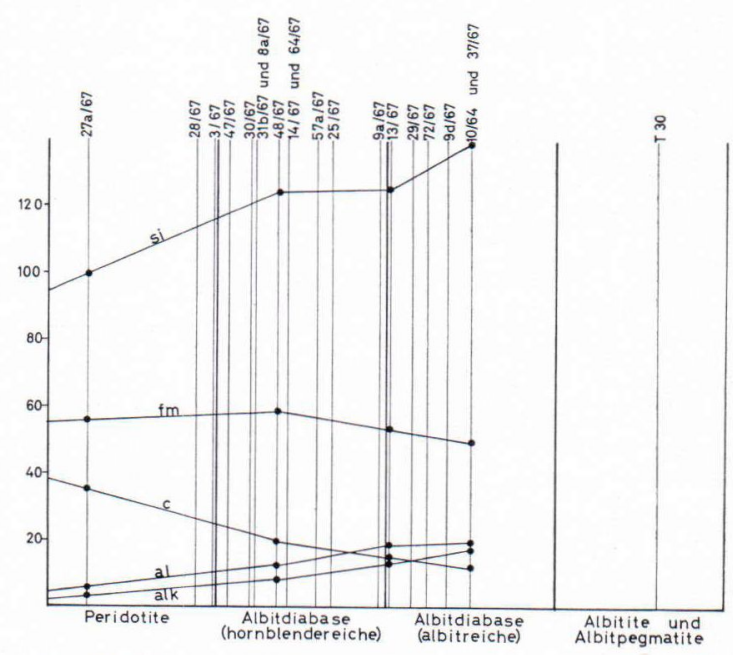

Abb. 13. Diagramm, in dem die analysierten Proben der spilitischen Assoziation nach ihren Niggliwerten angeordnet sind. Die Geraden mit schwarzen Punkten entsprechen den Werten in der Tabelle 8 und die anderen den Werten in der Tabelle 5. 


\section{TABelle 9.}

Cr-, Fe-, Ti-, Mn-, Co- und Cu-Gehalte der Metadiabase und der Gesteine der hypabyssischen Spilitassoziation im Koli-Kaltimogebiet. Anal. von A. Löfgren, P. Väänänen und R. Saikkonen in Geologinen tutkimuslaitos.

\begin{tabular}{|c|c|c|c|c|c|c|c|c|c|}
\hline \multirow{2}{*}{ Gesteinsart } & \multirow{2}{*}{ Probe und Lage } & \multicolumn{8}{|c|}{ Gewichtgehalte in $\%$} \\
\hline & & $\mathrm{Cr}$ & $\mathrm{Fe}$ & $\mathrm{Ti}$ & $\mathrm{Mn}$ & $\mathrm{V}$ & $\mathrm{Ni}$ & Co & $\mathrm{Cu}$ \\
\hline Uralitdiabas & $\begin{array}{r}1 / 67 x=503.10, y=6988.50 \\
12 / 67 x=493.55, y=6989.55 \\
21 / 67 x=496.60, y=6987.55 \\
23 / 67 x=494.30, y=6984.85\end{array}$ & $\begin{array}{l}0.03 \\
0.01\end{array}$ & $\begin{array}{l}8.33 \\
7.10\end{array}$ & $\begin{array}{l}0.53 \\
0.50 \\
0.62 \\
0.62 \\
\end{array}$ & $\begin{array}{l}0.13 \\
0.11 \\
0.16 \\
0.14\end{array}$ & $\begin{array}{l}0.042 \\
0.039 \\
0.044 \\
0.043\end{array}$ & $\begin{array}{l}0.019 \\
0.008 \\
0.016 \\
0.017\end{array}$ & $\begin{array}{l}0.0072 \\
0.0055 \\
0.0064 \\
0.0066\end{array}$ & $\begin{array}{l}0.16 \\
0.008 \\
0.026 \\
0.014 \\
\end{array}$ \\
\hline $\begin{array}{l}\text { Grünstein- } \\
\text { diabas }\end{array}$ & $\begin{array}{r}11 b / 67 x=492.70, y=6988.40 \\
10 / 67 x=490.15, y=6995.80 \\
19 a / 67 x=496.30, y=6989.00 \\
40 / 67 x=502.05, y=6977.55 \\
55 / 67 x=499.65, y=6980.20 \\
62 / 67 x=505.35, y=6974.80 \\
71 / 67 x=501.30, y=6975.00 \\
35 d / 67 x=498.65, y=6989.70 \\
101 / 64 x=501.25, y=6979.70\end{array}$ & & 10.48 & $\begin{array}{l}0.90 \\
0.84 \\
0.49 \\
0.62 \\
1.39 \\
0.46 \\
0.69 \\
1.62 \\
0.93 \\
\end{array}$ & $\begin{array}{l}0.15 \\
0.18 \\
0.17 \\
0.13 \\
0.18 \\
0.13 \\
0.16 \\
0.20 \\
0.15\end{array}$ & $\begin{array}{l}0.055 \\
0.059 \\
0.047 \\
0.038 \\
0.064 \\
0.038 \\
0.045 \\
0.125)\end{array}$ & $\begin{array}{l}0.006 \\
0.005 \\
0.016 \\
0.005 \\
0.007 \\
0.024 \\
0.007 \\
0.007\end{array}$ & $\begin{array}{l}0.0068 \\
0.0058 \\
0.0066 \\
0.0049 \\
0.0054 \\
0.0072 \\
0.0066 \\
0.0067\end{array}$ & $\begin{array}{l}0.028 \\
0.07 \\
0.009 \\
0.015 \\
0.012 \\
0.012 \\
0.033 \\
0.047\end{array}$ \\
\hline Peridotit & $\begin{array}{r}27 a / 67 x=398.95, y=6991.25 \\
28 / 67 x=498.95, y=6990.60 \\
3 / 67 x=491.90, y=6999.70\end{array}$ & 0.20 & 6.40 & $\begin{array}{l}0.54 \\
0.82 \\
0.65\end{array}$ & $\begin{array}{l}0.12 \\
0.16 \\
0.16\end{array}$ & $\begin{array}{l}0.029 \\
0.036 \\
0.030\end{array}$ & $\begin{array}{l}0.035 \\
0.023 \\
0.023\end{array}$ & $\begin{array}{l}0.0064 \\
0.023 \\
0.023\end{array}$ & $\begin{array}{l}0.010 \\
0.008 \\
0.011\end{array}$ \\
\hline $\begin{array}{l}\text { Hornblende- } \\
\text { reicher } \\
\text { Albitdiabas }\end{array}$ & $\begin{array}{r}47 / 67 x=501.65, y=6981.30 \\
30 / 67 x=499.70, y=6987.20 \\
31 b / 67 x=500.40, y=6986.20 \\
8 \mathrm{a} / 67 \mathrm{x}=493.15, y=6996.00 \\
48 / 67 \mathrm{x}=501.40, y=6980.25 \\
14 / 67 \mathrm{x}=495.65, y=6989.55 \\
64 / 67 \mathrm{x}=505.25, y=6974.50 \\
57 \mathrm{a} / 67 \mathrm{x}=497.80, y=6984.70 \\
25 / 67 \mathrm{x}=498.30, y=6991.35 \\
9 \mathrm{a} / 67 \mathrm{x}=490.65, y=6997.55 \\
\end{array}$ & 0.02 & 6.81 & $\begin{array}{l}1.44 \\
0.94 \\
1.28 \\
0.49 \\
1.04 \\
0.47 \\
1.56 \\
1.00 \\
1.35 \\
1.15 \\
\end{array}$ & $\begin{array}{l}0.19 \\
0.18 \\
0.12 \\
0.14 \\
0.18 \\
0.14 \\
0.16 \\
0.24 \\
0.21 \\
0.12\end{array}$ & $\begin{array}{l}0.054 \\
0.046 \\
0.048 \\
0.029 \\
0.088 \\
0.034 \\
0.069 \\
0.056 \\
0.061 \\
0.054\end{array}$ & $\begin{array}{l}0.006 \\
0.020 \\
0.022 \\
0.018 \\
0.020 \\
0.020 \\
0.014 \\
0.017 \\
0.003 \\
0.014\end{array}$ & $\begin{array}{l}0.0058 \\
0.020 \\
0.022 \\
0.0052 \\
0.0047 \\
0.0074 \\
0.0088 \\
0.017 \\
0.0030 \\
0.014 \\
\end{array}$ & $\begin{array}{l}0.022 \\
0.034 \\
0.041 \\
0.003 \\
0.002 \\
0.020 \\
0.039 \\
0.042 \\
0.041 \\
0.052 \\
\end{array}$ \\
\hline $\begin{array}{l}\text { Albitreicher } \\
\text { Albitdiabas }\end{array}$ & $\begin{array}{l}13 / 67 x=495.60, y=6989.50 \\
29 / 67 x=499.10, y=6987.75 \\
72 / 67 x=500.95, y=6976.55 \\
9 d / 67 x=490.65, y=6997.55 \\
10 / 64 x=506.80, y=6972.10 \\
37 / 64 x=496.70, y=6986.75\end{array}$ & 0.02 & $\begin{array}{l}11.29 \\
11.85\end{array}$ & $\begin{array}{l}1.15 \\
1.20 \\
1.84 \\
1.02 \\
1.37 \\
1.12 \\
\end{array}$ & $\begin{array}{l}0.13 \\
0.18 \\
0.26 \\
0.06 \\
0.10 \\
0.16\end{array}$ & $\begin{array}{l}0.051 \\
0.011 \\
0.048 \\
0.023 \\
0.043 \\
0.042\end{array}$ & $\begin{array}{r}0.010 \\
<0.001 \\
<0.001 \\
0.005 \\
0.001 \\
<0.001\end{array}$ & $\begin{array}{r}0.0058 \\
<0.0010 \\
0.0058 \\
0.0054 \\
0.0086 \\
<0.0010 \\
\end{array}$ & $\begin{array}{l}0.023 \\
0.030 \\
0.038 \\
0.007 \\
0.020 \\
0.045\end{array}$ \\
\hline Albitpegmatit & $\mathrm{T} 30 \mathrm{x}=500.70, \mathrm{y}=6980.60$ & & & 0,23 & 0,03 & 0,003 & 0,001 & 0,0010 & 0,001 \\
\hline $\begin{array}{c}\text { Albit-Karbo- } \\
\text { natgänge }\end{array}$ & $\begin{array}{r}35 b / 67 x=498.65, y=6986.70 \\
4 a / 67 x=491.90, y=6999.70 \\
7 / 67 x=491.90, y=6999.70 \\
6 a / 67 x=491.90, y=6999.70\end{array}$ & & & $\begin{array}{l}0.64 \\
1.68 \\
1.00 \\
0.10 \\
\end{array}$ & $\begin{array}{l}0.13 \\
0.24 \\
0.40 \\
0.44\end{array}$ & $\begin{array}{l}0.028 \\
0.130 \\
0.022 \\
0.006\end{array}$ & $\begin{array}{l}0.012 \\
0.075 \\
0.010 \\
0.004\end{array}$ & $\begin{array}{l}0.0063 \\
0.0110 \\
0.0050 \\
0.0015 \\
\end{array}$ & $\begin{aligned} & 0.020 \\
& 0.11 \\
& 0.015 \\
&< 0.001 \\
&\end{aligned}$ \\
\hline $\begin{array}{l}\text { Sulfidhaltige } \\
\text { Karbonat- } \\
\text { Quarzgänge }\end{array}$ & $\begin{array}{l}76 \mathrm{~b} / 67 \mathrm{x}=502.20, y=6979.50 \\
75 \mathrm{~b} / 67 \mathrm{x}=497.45, \mathrm{y}=6971.90 \\
52 \mathrm{~b} / 65 \mathrm{x}=500.65, \mathrm{y}=6982.15 \\
77 \mathrm{~b} / 67 \mathrm{x}=497.60, \mathrm{y}=6982.50\end{array}$ & & & $\begin{array}{l}0.07 \\
0.02 \\
0.70 \\
0.03 \\
\end{array}$ & $\begin{array}{l}0.00 \\
0.04 \\
0.08 \\
0.02\end{array}$ & $\begin{array}{r}0.002 \\
<0.001 \\
0.053 \\
0.001\end{array}$ & $\begin{array}{l}0.011 \\
0.004 \\
0.009 \\
0.110\end{array}$ & $\begin{aligned} & 0.025 \\
&< 0.0010 \\
& 0.0076 \\
& 0.045\end{aligned}$ & $\begin{array}{l}5.75 \\
2.43 \\
1.23 \\
1.20 \\
\end{array}$ \\
\hline
\end{tabular}


TABELLE 10.

Durchschnittswerte einzelner Gruppen der untersuchten tholeitischen und spilitischen Gesteine.

\begin{tabular}{|c|c|c|c|c|c|c|c|c|}
\hline Art der Probe & $\mathrm{Cr}$ & $\mathrm{Fe}$ & $\mathrm{Ti}$ & V & $\mathrm{Mn}$ & $\mathrm{Ni}$ & Co & $\mathrm{Cu}$ \\
\hline Uralitdiabase $\ldots \ldots \ldots \ldots \ldots \ldots \ldots$ & 0.02 & 7.72 & 0.57 & 0.042 & 0.14 & 0.015 & 0.0064 & 0.016 \\
\hline Grünsteindiabase $\ldots \ldots \ldots \ldots \ldots$ & - & - & 0.88 & 0.049 & 0.16 & 0.010 & 0.0063 & 0.028 \\
\hline Peridotite $\ldots \ldots \ldots \ldots \ldots \ldots \ldots \ldots$ & 0.20 & 6.40 & 0.67 & 0.032 & 0.15 & 0.027 & 0.017 & 0.010 \\
\hline Hornblendereiche Albitdiabase ... & 0.02 & 6.81 & 1.07 & 0.054 & 0.17 & 0.015 & 0.011 & 0.030 \\
\hline Hornblendearme Albitdiabase ... & 0.02 & 11.57 & 1. 28 & 0.036 & 0.15 & 0.003 & 0.005 & 0.027 \\
\hline Albitpegmatit $\ldots \ldots \ldots \ldots \ldots \ldots$ & - & - & 0.23 & 0.003 & 0.03 & 0.001 & 0.001 & 0.001 \\
\hline Albit-Karbonatgänge $\quad \ldots \ldots \ldots \ldots$ & - & - & 0.86 & 0.046 & 0.30 & 0.025 & 0.006 & 0.004 \\
\hline Kieshaltige Quarz-Karbonatgänge & - & 一 & 0.21 & 0.014 & 0.03 & 0.035 & 0.020 & 2.65 \\
\hline
\end{tabular}

TABELLE 11.

Verteilung der schweren Metalle in verschiedene Mineralien in den Gesteinsarten der tholeitischen und spilitischen Assoziation des Koli-Kaltimogebiets nach den Elektronensondebestimmungen, die im Labor der Outokumpu A.G. gemacht worden sind.

\begin{tabular}{|c|c|c|c|c|c|c|c|c|c|c|c|c|}
\hline \multicolumn{13}{|c|}{ Gesteins art } \\
\hline \multirow[b]{2}{*}{ Metall } & \multirow[b]{2}{*}{ Mineral } & \multicolumn{2}{|c|}{ Uralitdiabas } & \multicolumn{2}{|c|}{ Grünsteindiabas } & \multicolumn{2}{|c|}{ Peridotit } & \multicolumn{2}{|c|}{$\begin{array}{c}\text { Hornblendereicher } \\
\text { Albitdiabas }\end{array}$} & \multicolumn{3}{|c|}{$\begin{array}{l}\text { Albitreicher } \\
\text { Albitdiabas }\end{array}$} \\
\hline & & $1 / 67$ & $12 / 67$ & $62 / 67$ & $71 / 67$ & $27 \mathrm{a} / 67$ & $3 / 67$ & $48 / 67$ & $13 / 67$ & $37 / 67$ & $10 / 64$ & $\mathrm{E} / \mathrm{p}-5$ \\
\hline $\mathrm{Cr}$ & 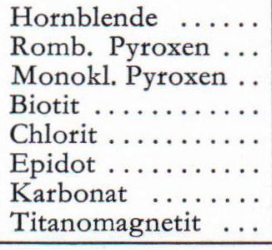 & $\begin{array}{l}0.01 \\
0.004 \\
0.05 \\
- \\
- \\
- \\
0.21\end{array}$ & $\begin{array}{r}0.01 \\
- \\
0.002 \\
- \\
- \\
0.01\end{array}$ & $\begin{array}{r}0.04 \\
0 . \overline{07} \\
\overline{-0} \\
0.01 \\
0.03 \\
-\overline{03}\end{array}$ & $\begin{array}{r}0.04 \\
- \\
- \\
0.00 \\
0.02 \\
-\overline{0} \\
0.00\end{array}$ & $\begin{array}{l}0.09 \\
0.25 \\
- \\
- \\
- \\
-\end{array}$ & $\begin{array}{r}0.01 \\
-\overline{00} \\
- \\
- \\
0.00 \\
0.00 \\
-\end{array}$ & $\begin{array}{r}0.03 \\
- \\
0.01 \\
- \\
- \\
0.02\end{array}$ & $\begin{array}{r}0.005 \\
- \\
0.03 \\
- \\
- \\
0.04\end{array}$ & $\begin{array}{r}0.00 \\
- \\
- \\
- \\
- \\
0.01\end{array}$ & $\begin{array}{r}0.002 \\
- \\
0.005 \\
- \\
- \\
0.01\end{array}$ & $\begin{array}{l}0.01 \\
- \\
- \\
0.01 \\
0.01 \\
0 . \overline{01}\end{array}$ \\
\hline $\mathrm{Fe}$ & 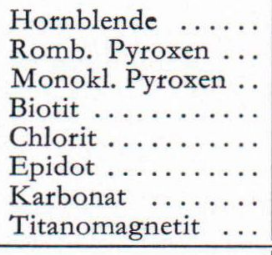 & $\begin{array}{r}13.9 \\
18.4 \\
8.5 \\
- \\
- \\
- \\
-\end{array}$ & $\begin{array}{r}11.1 \\
17.1 \\
- \\
- \\
-\end{array}$ & $\begin{array}{r}8.05 \\
-\overline{94} \\
-\overline{87} \\
12.8 .46 \\
-\overline{98} \\
\end{array}$ & $\begin{array}{r}11.72 \\
- \\
-\overline{7} \\
71.01 \\
-\overline{42} \\
\end{array}$ & 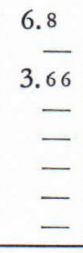 & $\begin{array}{r}9.11 \\
5.57 \\
- \\
9.65 \\
0.46 \\
\end{array}$ & $\begin{array}{r}7.5 \\
10.6 \\
- \\
- \\
-\end{array}$ & $\begin{array}{r}8.3 \\
11.4 \\
- \\
- \\
-\end{array}$ & $\begin{array}{r}14.15 \\
- \\
- \\
- \\
5 \\
- \\
\end{array}$ & $\begin{array}{r}11.6 \\
- \\
14.2 \\
- \\
- \\
-\end{array}$ & $\begin{array}{r}12.03 \\
- \\
- \\
24 . \overline{38} \\
10.92 \\
65 . \overline{80} \\
\end{array}$ \\
\hline Ti & 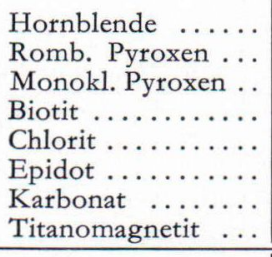 & $\begin{array}{r}0.14 \\
0.22 \\
0.16 \\
- \\
- \\
- \\
-\end{array}$ & $\begin{array}{r}0.11 \\
- \\
0.75 \\
- \\
22.4\end{array}$ & $\begin{array}{r}0.04 \\
-\overline{22} \\
-\overline{00} \\
0.06 \\
32.41 \\
\end{array}$ & $\begin{array}{r}0.10 \\
-\overline{-} \\
0.03 \\
0.09 \\
32.61 \\
\end{array}$ & $\begin{array}{r}0.21 \\
0.17 \\
- \\
- \\
- \\
-\end{array}$ & $\begin{array}{r}0.06 \\
0.06 \\
- \\
0.02 \\
0.00 \\
-\end{array}$ & $\begin{array}{r}0.09 \\
- \\
0.60 \\
- \\
- \\
5.0\end{array}$ & $\begin{array}{r}0.01 \\
- \\
0.73 \\
- \\
- \\
3.65\end{array}$ & $\begin{array}{r}0.16 \\
- \\
- \\
- \\
- \\
7.52\end{array}$ & $\begin{array}{r}0.15 \\
- \\
0.73 \\
- \\
- \\
2.19\end{array}$ & $\begin{array}{l}0.08 \\
- \\
- \\
0.01 \\
0.51 \\
0 . \overline{23} \\
\end{array}$ \\
\hline V & 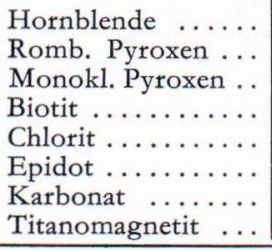 & $\begin{array}{r}0.02 \\
0.02 \\
0.05 \\
- \\
- \\
- \\
- \\
\end{array}$ & $\begin{array}{l}0.04 \\
\frac{-}{-} \\
0.05 \\
- \\
- \\
0.66\end{array}$ & $\begin{array}{r}0.03 \\
-\overline{05} \\
-\overline{0} \\
0.01 \\
0.05 \\
-\overline{26}\end{array}$ & $\begin{array}{r}0.04 \\
- \\
- \\
0.06 \\
0.04 \\
-\overline{27} \\
\end{array}$ & $\begin{array}{r}0.02 \\
- \\
0.02 \\
- \\
- \\
- \\
-\end{array}$ & $\begin{array}{r}0.04 \\
-\overline{-} \\
0.02 \\
- \\
-\overline{02} \\
0.02\end{array}$ & $\begin{array}{r}0.09 \\
- \\
0.13 \\
- \\
- \\
0.46\end{array}$ & $\begin{array}{r}0.02 \\
- \\
0.007 \\
- \\
- \\
0.36\end{array}$ & $\begin{array}{r}0.04 \\
- \\
- \\
- \\
- \\
- \\
0.25\end{array}$ & $\begin{array}{r}0.04 \\
- \\
0.05 \\
- \\
- \\
0.30\end{array}$ & $\begin{array}{r}0.03 \\
- \\
- \\
-\overline{03} \\
0.01 \\
-\overline{22}\end{array}$ \\
\hline
\end{tabular}


Tabelle 11. Forts.

\begin{tabular}{|c|c|c|c|c|c|c|c|c|c|c|c|c|}
\hline $\mathrm{Mn}$ & 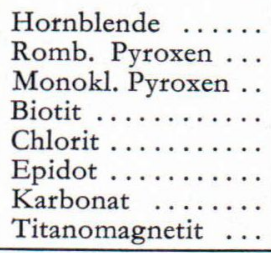 & $\begin{array}{r}0.18 \\
0.29 \\
0.17 \\
- \\
- \\
- \\
0.18\end{array}$ & $\begin{array}{r}0.16 \\
- \\
0.11 \\
= \\
= \\
0.52\end{array}$ & $\begin{array}{l}0.15 \\
\overline{-} \\
0.11 \\
0 . \overline{23} \\
0.16 \\
\overline{2.10}\end{array}$ & $\begin{array}{r}0.19 \\
- \\
- \\
0.04 \\
0.20 \\
\overline{2.89}\end{array}$ & $\begin{array}{r}0.12 \\
0.07 \\
- \\
= \\
= \\
-\end{array}$ & $\begin{array}{r}0.20 \\
-\overline{13} \\
- \\
- \\
0.07 \\
0.19 \\
-\end{array}$ & $\begin{array}{r}0.23 \\
- \\
0.09 \\
- \\
= \\
0.26\end{array}$ & $\begin{array}{r}0.31 \\
-\overline{-} \\
0.15 \\
- \\
- \\
0.05\end{array}$ & $\begin{array}{r}0.22 \\
\text { - } \\
- \\
- \\
\overline{-} \\
0.47\end{array}$ & $\begin{array}{r}0.33 \\
- \\
0.21 \\
- \\
- \\
0.06\end{array}$ & $\begin{array}{l}0.23 \\
- \\
-\bar{Z} \\
0.29 \\
0.25 \\
0 . \overline{03}\end{array}$ \\
\hline $\mathrm{Ni}$ & 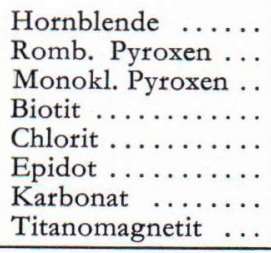 & $\begin{array}{r}0.02 \\
0.02 \\
0.02 \\
- \\
- \\
- \\
0.02\end{array}$ & $\begin{array}{r}0.01 \\
- \\
\overline{-} \\
0.01 \\
- \\
\overline{-} \\
0.006\end{array}$ & $\begin{array}{l}- \\
- \\
- \\
- \\
-\end{array}$ & $\begin{array}{l}- \\
\bar{z} \\
\overline{-} \\
\overline{-} \\
-\end{array}$ & $\begin{array}{r}0.02 \\
0.03 \\
- \\
- \\
- \\
-\end{array}$ & $\begin{array}{l}- \\
- \\
- \\
- \\
-\end{array}$ & $\begin{array}{r}0.01 \\
\overline{-} \\
0.02 \\
\overline{-} \\
\overline{-} \\
0.039\end{array}$ & $\begin{array}{l}0.01 \\
\overline{-} \\
0.02 \\
= \\
\overline{-} \\
0.005\end{array}$ & - & $\begin{array}{r}0.002 \\
\overline{-} \\
0.002 \\
\overline{-} \\
\bar{z} \\
0.008\end{array}$ & $\begin{array}{l}- \\
= \\
\text { I }\end{array}$ \\
\hline Co & 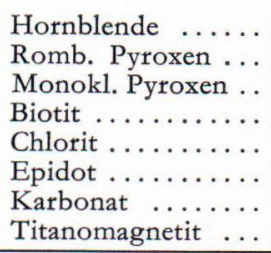 & $\begin{array}{l}0.01 \\
0.01 \\
0.004 \\
- \\
- \\
- \\
0.01\end{array}$ & $\begin{array}{r}0.003 \\
\overline{-} \\
0.005 \\
- \\
\overline{-} \\
0.004\end{array}$ & $\begin{array}{l}- \\
- \\
- \\
- \\
-\end{array}$ & $\begin{array}{l}= \\
= \\
= \\
= \\
=\end{array}$ & $\begin{array}{r}0.002 \\
-0.003 \\
= \\
= \\
=\end{array}$ & $\begin{array}{l}- \\
- \\
- \\
- \\
-\end{array}$ & $\begin{array}{r}0.004 \\
\overline{-} \\
0.004 \\
- \\
\overline{-} \\
0.01\end{array}$ & $\begin{array}{r}0.004 \\
- \\
0.002 \\
- \\
= \\
- \\
0.005\end{array}$ & $\begin{array}{l}= \\
\bar{z} \\
= \\
=\end{array}$ & $\begin{array}{r}0.000 \\
- \\
0.002 \\
= \\
= \\
0.005\end{array}$ & $\begin{array}{l}= \\
I \\
I \\
I\end{array}$ \\
\hline $\mathrm{Cu}$ & 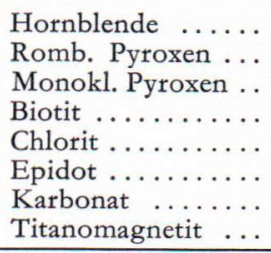 & $\begin{array}{r}0.01 \\
0.01 \\
0.01 \\
- \\
- \\
- \\
0.02\end{array}$ & $\begin{array}{r}0.01 \\
- \\
0.01 \\
- \\
\overline{-} \\
0.009\end{array}$ & $\begin{array}{l}- \\
- \\
- \\
- \\
-\end{array}$ & $\begin{array}{l}= \\
= \\
= \\
= \\
=\end{array}$ & $\begin{array}{r}0.02 \\
-\overline{-} \\
0.01 \\
- \\
- \\
-\end{array}$ & $\begin{array}{l}- \\
- \\
- \\
- \\
-\end{array}$ & $\begin{array}{r}0.01 \\
\text { - } \\
0.02 \\
= \\
= \\
0.022\end{array}$ & $\begin{array}{r}0.01 \\
- \\
0.01 \\
= \\
= \\
0.006\end{array}$ & $\begin{array}{l}- \\
- \\
- \\
- \\
-\end{array}$ & $\begin{array}{r}0.01 \\
- \\
0.02 \\
= \\
= \\
0.02\end{array}$ & $\begin{array}{l}\text { I } \\
\text { I } \\
\text { I }\end{array}$ \\
\hline
\end{tabular}

spilitischen Differentiationsserie von Peridotiten über hornblendereiche Albitdiabase und albitreiche Albitdiabase bis zu Albitpegmatiten sowie Albit-Karbonat- und kieshaltigen QuarzKarbonatgängen. Die hypabyssische spilitische Differentiationsserie ist in der Abbildung 13 dargestellt, die auf Grund der Niggeliwerte (s. Tab. 8) und des Mineralbestands der analysierten Proben (s. Tab. 5) gezeichnet worden ist. Diese Abbildung ist die Grundlage zu den Abbildungen 14, 15, 16 und 17, welche die Verteilung der Elemente in den Gesteinen des initialen Magmatismus im Koli-Kaltimogebiet veranschaulichen. Auf Grund der angeführten Daten wird das Benehmen jedes einzelnen Elements im folgenden näher betrachtet.

\section{Chrom}

Es gibt nur fünf Totalbestimmungen für das Chrom. Von diesen Bestimmungen sind zwei von Uralitdiabasen, zwei von Peridotiten, eine von hornblendereichen Albitdiabasen und eine von albitreichen Albitdiabasen gemacht worden. Die Analysen zeigen (Abb. 14 und 16), dass das Chrom sich stark mit Peridotiten angereichert hat und sein Gehalt $0.2 \%$ beträgt. In den übrigen Gesteinsgruppen beträgt der Cr-Gehalt nur ein Zehntel von dem Cr-Gehalt der Peridotite.

In der erzmikroskopischen Untersuchung hat man keine Chrommineralien gefunden, weshalb man annehmen kann, dass das Chrom in den 

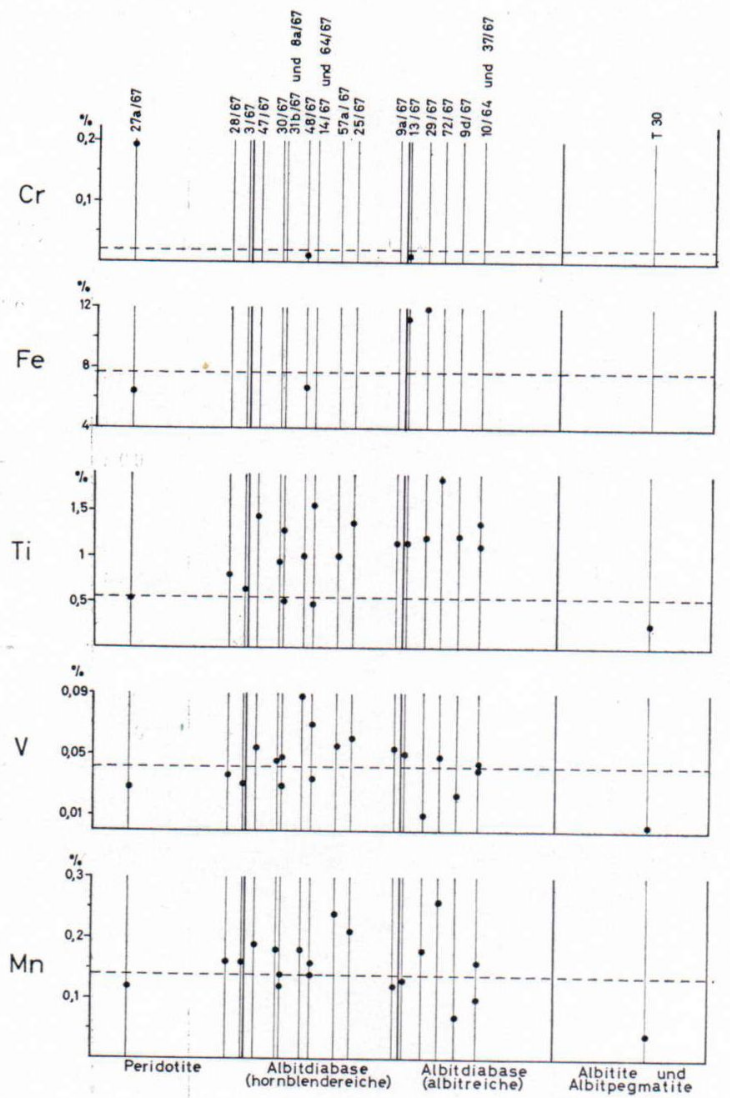

Abb. 14. Der Wechsel des Cr, Fe, Ti, V und Mn in der spilitischen Assoziation. Die schwarzen Punkte entsprechen den Werten in der Tabelle 9 und die gebrochene Linie den in der Tabelle 10 dargestellten durchschnittlichen Werten der Uralitdiabase.

Gittern der Silikate getarnt ist. Nach den mikroanalysatorischen Bestimmungen ist der grösste Cr-Gehalt in den Pyroxenen zu finden (s. Tab. 11). In kleinerem Masse sind auch Titanomagnetit, Hornblende, Biotit, Chlorit und Epidot Cr-haltig. Der Ionenradius des Chroms $\left(\mathrm{Cr}^{3+}=0.63 \AA\right)$ gleicht sehr demjenigen des dreiwertigen Eisens $\left(\mathrm{Fe}^{3+}=0.64 \AA\right)$, des vierwertigen Titans ( $\left.\mathrm{Ti}{ }^{4+}=0.68 \AA\right)$, des Magnesiums $\left(\mathrm{Mg}^{2+}=0.66 \AA\right)$ und des $\mathrm{Alu-}$ miniums $\left(\mathrm{Al}^{3+}=0.51 \AA\right.$ ), so dass das Chrom wahrscheinlich an ihrer Stelle in den genannten Mineralien auftritt.
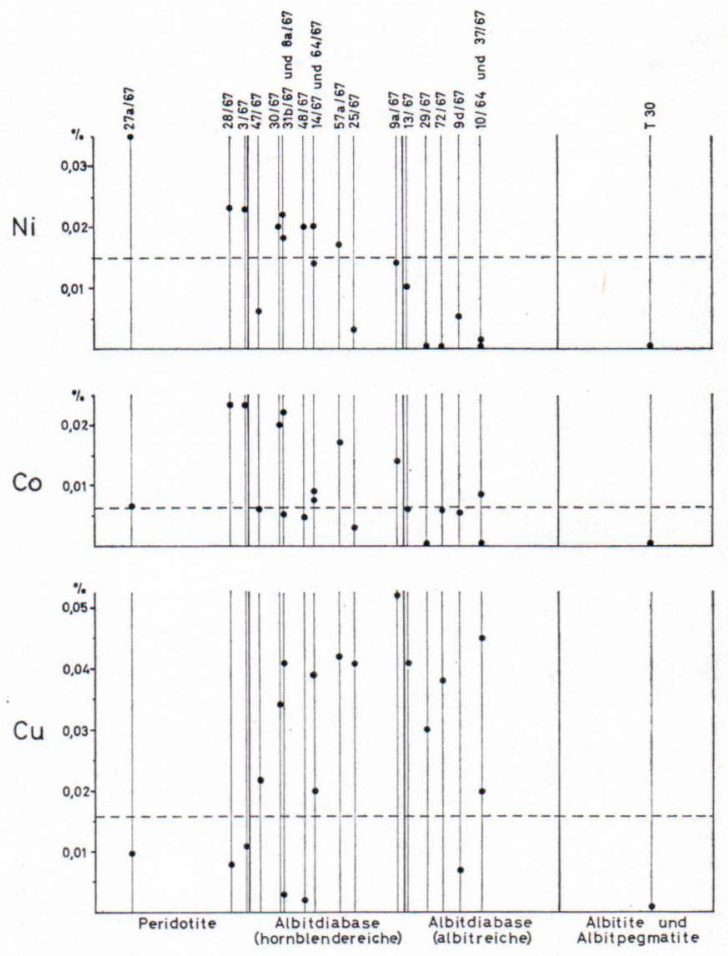

Abb. 15. Der Wechsel des Ni, Co und $\mathrm{Cu}$ in der spilitischen Assoziation. Die schwarzen Punkte entsprechen den Werten in der Tabelle 9 und die gebrochene Linie den in der Tabelle 10 dargestellten durchschnittlichen Werten der Uralitdiabase.

Der Chromgehalt der basischen Gänge des Gebiets entspricht gut dem durchschnittlichen Chromgehalt der Gabbros und der Basalte, der nach Goldschmidt (1954) $0.01-0.04 \%$, nach Vinogradov (1956) $0.02 \%$ und nach Turekian \& Carr (1960) $0.017 \%$ beträgt. In diesem Zusammenhang sei vergleichsweise der Chromgehalt z. B. des Karroo-Dolorits erwähnt, der nach Walker \& Polderwaart (1949) $0.028 \%$ beträgt.

\section{Eisen}

Für das Eisen sind nur sieben Totalbestimmungen gemacht worden: drei von den 

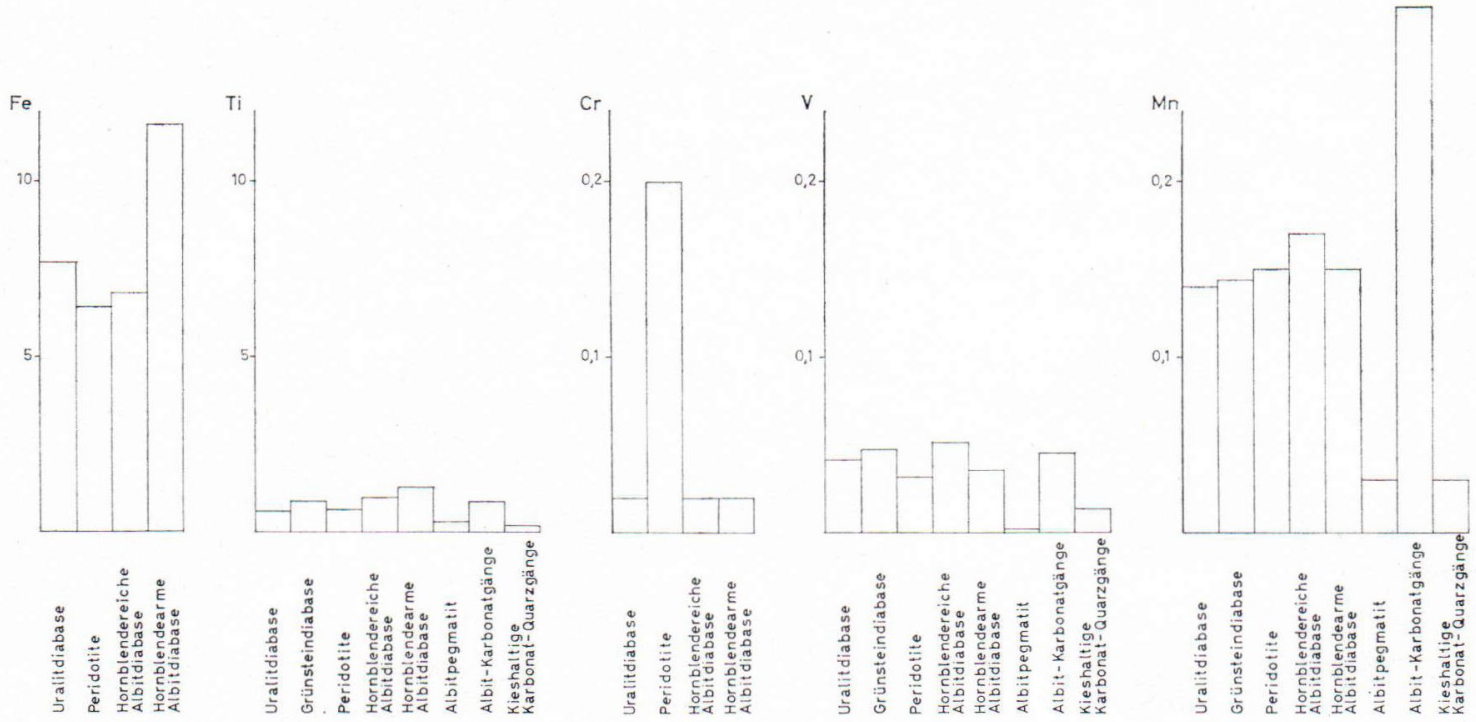

Abb. 16. Der durchschnittliche Wechsel des Cr, Fe, Ti, V und Mn in den Gesteinen des initielen Magmatismus im Koli-Kaltimogeobiet.

Metadiabasen und vier von den Gesteinen der spilitischen Assoziation. Nach den Analysen beträgt der Eisengehalt der Metadiabase 7.11$10.48 \%$ Er verteilt sich (s. Tab. 11) hauptsächlich auf Hornblende, rombischen und monoklinen Pyroxen, Epidot, Titanomagnetit und Biotit. In der spilitischen Gesteinsassoziation variiert die Menge des Eisens von $6.40 \%$ bis $11.85 \%$, und zwar so, dass sie am kleinsten in den Peridotiten und am grössten in den Albitdiabasen ist, die nahe den Albititen sind (s. Abb. 14). In den Peridotiten und den hornblendereichen Albitdiabasen ist das Eisen hauptsächlich mit den Pyroxenen und mit der Hornblende verbunden. Der Eisengehalt beider dieser Mineralien nimmt gegen die albitreiche Gesteine zu, dabei kommt

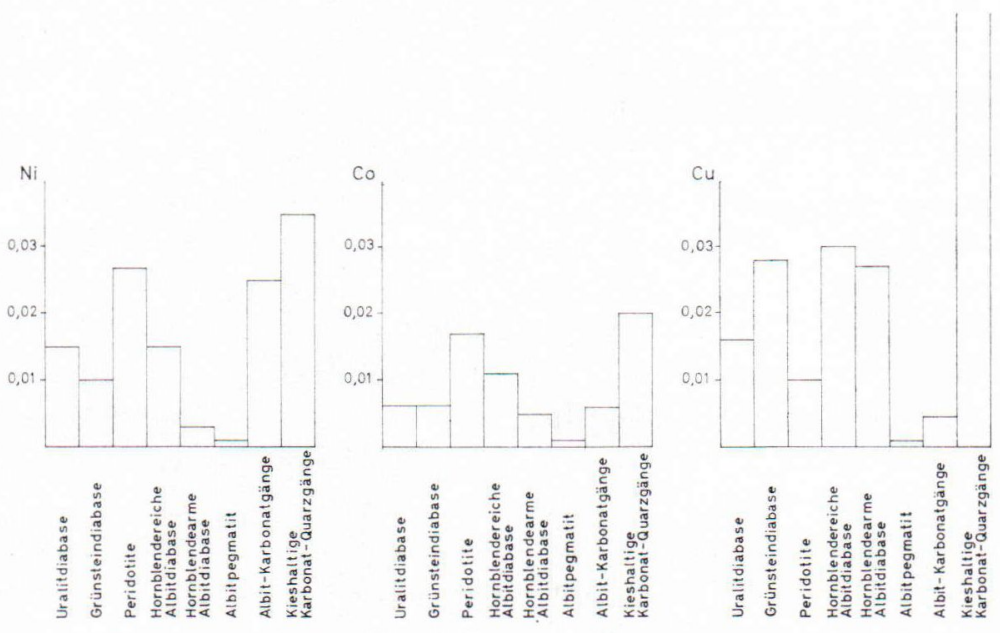

Abb. 17. Der durchschnittliche Wechsel des Ni, Co und $\mathrm{Cu}$ in den Gesteinen des initialen Magmatismus im Koli-Kaltimogebiet. 


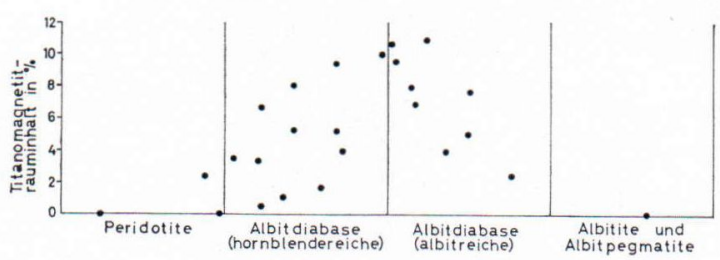

Abb. 18. Der Wechsel des Magnetits in der spilitischen Assoziation.

auch Titanomagnetit vor, dessen Menge in den albitreichen Albitdiabasen $10 \%$ beträgt (s. Abb. 18).

Titan

Ähnlich wie das Eisen kommt das Titan vor, das in 41 Proben analysiert worden ist. Die analysierten Probenvertreten alle Typen sowohl unter den Metadiabasen als auch in der spilitischen Differentiationsserie.

In den Uralitdiabasen wechselt der Titangehalt relativ wenig, von $0.50 \%$ bis $0.62 \%$. Im Vergleich zu ihnen weisen die Grünsteindiabase eine grössere Variation in dem Titangehalt auf. Auch der durchschnittliche Titangehalt der Grünsteindiabase ist ein wening grösser. Der Hauptteil des Titans sowohl in den Uralit- als auch in den Grünsteindiabasen hat sich in Ilmenit und in Titanit konzentriert, und das Schwanken des Titangehalts liegt zunächst an den Varianten dieser Mineralien.

In den Gesteinen der spilitischen Assoziation variiert der Titangehalt zwischen 0.47 und $1.84 \%$. Im Durchschnitt enthalten die Peridotite Titan am wenigsten und die reichlich Albit führenden Albitdiabase am meisten. Führt das Gestein reichlich Karbonate, nimmt der Titangehalt im allgemeinen ab (s. Proben 8a/67 und $14 / 67$ in der Tab. 9). Das Titan ist einigermassen in den Gittern der Silikate getarnt. Vor allem der Biotit und der Epidot können sehr titanhaltig sein. Dagegen ist der Titangehalt der Pyroxene und des Amphibols niedrig. Z. B. der Titangehalt der Hornblende variiert zwischen $0.01 \%$ und $0.21 \%$. Es können keine deutlichen Unterschiede in der Titanmenge zwischen verschiedenen Differentiaten desselben Minerals festgestellt werden, und die Zunahme des Titans in den albitreichen Gesteinen liegt einerseits an der Zunahme des Titanomagnetits, anderseits an derjenigen des Biotits und Epidots.

Der Titangehalt der Karbonatgänge schwankt sehr. Im allgemeinen ist er niedrig in den mittleren Teilen der Gänge, steigt aber nach den Rändern zu, wo der hohe Titangehalt wahrscheinlich ein Relikt des verdrängten Wirtsgesteins ist, denn hier kommt der Titanomagnetit zusammen mit Leukoxen in Form zerfressener Körner reichlich vor.

\section{Vanadin}

Die Totalbestimmungen des Vanadins belaufen sich insgesamt auf 40 . Sie verteilen sich auf folgende Weise auf die verschiedenen Gesteinsarten: 4 Bestimmungen sind über die Uralitdiabse, 8 über die Grünsteindiabase, 3 über die Peridotite, 10 über die hornblendereichen Albitdiabase, 6 über die albitreichen Albitdiabase, 1 über die Albitpegmatite und 8 über die pneumatolytischen und hydrothermalen Gangformationen gemacht worden.

Der Vanadingehalt der Uralitdiabase schwankt zwischen $0.039 \%$ und $0.044 \%$ mit einem Durchschnittsgehalt von $0.042 \%$. In den Grünsteindiabasen variiert er von $0.038 \%$ bis $0.064 \%$. Eine titanomagnetitreiche Probe, 35d/67, enthält sogar $0.125 \%$ V. Der durchschnittliche Gehalt der Grünsteindiabase beträgt $0.049 \% \mathrm{~V}$, ausser der obenerwähnten Probe.

In den Gesteinen der spilitischen Assoziation ist eine deutliche Verteilung des Vanadins auf verschiedene Differentiate $\mathrm{zu}$ sehen. In den Peridotiten beträgt der Vanadingehalt im Durchschnitt $0.032 \%$, ist also deutlich geringer als derjenige der Uralit- und Grünsteindiabase. Nach den hornblendereichen Albitdiabasen zu wächst der Vanadingehalt stark: Der durch- 
schnittliche Gehalt beträgt hier $0.054 \%$. Die Proben, deren Vanadingehalt darunter liegt, enthalten ausnahmslos Karbonate. Weiter nach den albitreichen Gesteinen $\mathrm{zu}$ in der Differentiationsserie beginnt der Vanadingehalt abzunehmen. Der durchschnittliche Wert der albitreichen Albitdiabase beträgt $0.036 \% \mathrm{~V}$. Auch der Vanadingehalt der Albitpegmatite sowie der pneumatolytischen und hydrothermalen Gänge ist im allgemeinen niedrig. Nur in solchen Proben, die aus den Rändern der Gänge stammen und die aus metasomatisch umgewandeltem Nebengestein bestehen, kann der Vanadingehalt wie der Titangehalt bedeutend hoch sein.

Das Vanadin bildet kaine eigenen Mineralien, sondern ist in dem Gitter der Hornblende, der Pyroxene, des Biotits, Chlorits, Epidots und vor allem des Titanomagnetits getarnt und ersetzt Eisen, Aluminium und möglicherweise auch Titanium, denen es in Hinsicht auf seine Iongrösse gleicht. Am grössten ist der Vanadingehalt der Mineralien in den hornblendereichen Albitdiabasen. Vor allem kommt dies beim Vanadingehalt des Titanomagnetits zum Vorschein, in dessen Magnetitkomponente das Vanadin zum grössten Teil eingebaut ist. Die Menge des Titanomagnetits ist am grössten in den albitreichen Albitdiabasen. Jedoch enthalten sie weniger Vanadin als die hornblendereichen Typen.

Wenn man den Vanadingehalt sowohl der Metadiabase als auch der spilitischen Gesteine des Gebiets mit demjenigen der Basalte in anderen Gebieten vergleicht, kann man feststellen, dass die betreffenden Gesteine 2-3 mal so viel Vanadin enthalten wie die Basalte im Durchschnitt (vgl. Goldschmit 1954, Vinogradov 1953, Agiorgitis 1968). Es soll auch hier vergleichsweise erwähnt werden, dass Dekkanbasalte im Durchschnitt $0.008 \%$ (Sinha und Karkare 1964), die Karroodolerite $0.015 \%$ (Walker \& Poldervaart 1949) sowie einige basaltische Gesteine im mittleren und südlichen Europa $0.0194 \%$ (Agiorgitis 1968) Vanadin enthalten. Ungefähr gleich hoch wie in den Gesteinen des Koli-Kaltimogebiets ist der Vanadingehalt in den in Kamtschatka und auf den Kurilen auftretenden basaltischen Gesteinen (vgl. Markhinin \& Sapozhikova 1962).

\section{Mangan}

Über das Mangan sind in allem 41 Totalbestimmungen gemacht worden: 4 über die Uralitdiabase, 9 über die Grünsteindiabase, 3 über die Peridotite, 10 über die hornblendereichen Albitdiabase, 7 über die albitreichen Albitdiabase, 1 über die Alpitpegmatite sowie 8 über die pneumatolytischen und hydrothermalen Gänge.

In Hinsicht auf den Mangangehalt gleichen die Uralitdiabase und die Grünsteindiabase einander ungefähr, und sie enthalten im Durchschnitt $0.15 \%$ Mn. In der spilitischen Assoziation ist die Verteilung des Mangans unter die verschiedenen Gesteinsarten deutlich, und zwar so, dass das Mangan am reichlichsten in den hornblendereichen Albitdiabasen vorkommt, während die Peridotite und die albitreichen Albitdiabase arm an Mangan sind. In den Albitpegmatiten sowie in den kieshaltigen Quarz-Karbonatgängen wird Mangan nicht nennenswert angetroffen. Die Albit-Karbonatgänge können dagegen bedeutend manganhaltig sein.

Diese Verteilung des Mangans unter die verschiedenen Gesteinsarten ist mit Rücksicht auf die Verteilung des Mangans unter die verschiedenen Mineralien zu verstehen. Das Mangan bildet keine eigenen Mineralien, sondern ist in den Gittern der anderen Mineralien, vor allem der Hornblende, des rombischen Pyroxens und der Ilmenitkomponente des Titanomagnetits getarnt (s. Tab. 11). Aus den Analysendaten lässt sich feststellen, dass der Mangangehalt der verschiedenen Mineralien nach den späteren Differentiaten zu wächst und der hohe Mangangehalt der hornblendereichen Albitdiabase auf die grosse Menge der Hornblende zurückzuführen ist. 


\section{Nickel}

Über das Nickel sind in allem 40 Totalbestimmungen gemacht worden: 4 über die Uralitdiabase, 8 über die Grünsteindiabase, 3 über die Peridotite, 10 über die hornblendereichen Albitdiabase, 6 über die albitreichen Albitdiabasen, 1 über die Albitpegmatite sowie 8 über die pneumatolytischen und hydrothermalen Gänge.

Der Nickelgehalt der Metadiabase wechselt zwischen $0.005 \%$ und $0.024 \%$. Dieser Wechsel ist in den Grünsteindiabasen etwas grösser als in den Uralitdiabasen, was darauf hindeutet, dass das Nickel in der Metamorphose mobil geworden ist. Der duchschnittliche Nickelgehalt der Uralitdiabase beträgt $0.015 \%$. Dieses Ergebnis entspricht gut dem durchschnittlichen Nickelgehalt der normalen Basalte, der nach Goldschmidt (1954) $0.016 \%$, nach Carr \& Turekian (1961) $0.013 \%$, nach Vinogradov (1962) $0.016 \%$ und nach Agiorgitis (1968) $0.0145 \%$ beträgt. Der durchschnittliche Nickelgehalt der Grünsteindiabase, der $0.010 \%$ ist, weicht dagegen von den obengenannten Daten ab.

In der spilitischen Gesteinsassoziation ist das Nickel in einer bestimmten Weise unter die verschiedenen Gesteinsasrten und unter ihre Mineralien verteilt. In den Peridotiten, die im Durchschnitt $0.027 \% \mathrm{Ni}$ enthalten, ist der Nickelgehalt am grössten und nimmt nach den albitreicheren Gesteinsarten zu ab. Die hornblendereichen Albitdiabase enthalten durchschnittlich $0.015 \% \mathrm{Ni}$, die albitreichen Albitdiabase nur $0.003 \%$ und die Albitpegmatite $0.001 \%$. In den pneumatolytischen und hydrothermalen Gängen wechselt der Nickelgehalt stark. Die durchschnittlichen Werte sind hier wieder grösser.

Das Nickel ist nach seiner Iongrösse $\left(\mathrm{Ni}^{2+}=\right.$ $0.69 \AA)$ dem Magnesium $\left(\mathrm{Mg}^{2+}=0.66 \AA\right)$ sowie dem zwei- und dreiwertigen Eisen $\left(\mathrm{Fe}^{2+}=\right.$ $0.74 \AA, \mathrm{Fe}^{3+}=0.64 \AA$ ) sehr ähnlich, was zu der Annahme führt, dass das Nickel sie in den Pyroxenen, der Hornblende und dem Biotit vertritt, mit denen das Nickel der Gesteine zum grössten Teil verbunden ist (s. Tab. 11). Jedenfalls ist der Nickelgehalt dieser Mineralien grösser als der Gesamtgehalt des ganzen Gesteines.

\section{Kobalt}

Über das Kobalt sind 40 Totalbestimmungen gemacht worden. Diese Analysen verteilen sich unter die verschiedenen Gesteinsarten auf dieselbe Weise wie diejenigen des Nickels.

Der Kobaltgehalt der Metadiabase variiert zwischen $0.0049 \%$ und $0.0072 \%$. Es gibt keinen nennenswerten Unterschied zwischen dem Kobaltgehalt der Uralitdiabase und dem der Grünsteindiabase. Die Uralitdiabase enthalten im Durchschnitt $0.0064 \%$ und die Grünsteindiabase $0.0063 \%$ Co. Die Daten entsprechen gut dem Kobaltgehalt der Basalte, der nach Goldschmidt (1954) $0.0080 \%$, nach Carr und Turekian (1959) $0.0048 \%$, nach Vinogradov (1962) $0.0045 \%$ und nach Agiorgitis $0.0037 \%$ beträgt.

In der spilitischen Gesteinsassoziation ist das Kobalt am reichlichsten in den Peridotiten vorhanden, die im Durchschnitt $0.017 \%$ Co enthalten. Nach den albitreicheren Typen zu nimmt er ab. Die hornblendereichen Albitdiabase enthalten im Durchschnitt $0.011 \%$, die albitreichen Albitdiabase $0.005 \%$ und die Albitpegmatite $0.001 \%$ Co. Obgleich die Abnahme des Kobalts in den albitreicheren Typen deutlich ist, zeigt sie jedoch keine so steile Kurve wie die Abnahme des Nickels. Die albitreichen Albitdiabase enthalten sogar mehr Kobalt als Nickel. Wie das Nickel hat sich auch das Kobalt in den kieshaltigen hydrothermalen Gängen angereichert.

Das Kobalt gleicht, was die Iongrösse $\left(\mathrm{Co}^{2+}=\right.$ $0.72 \AA, \mathrm{Co}^{3+}=0.63 \AA$ ) betrifft, sehr dem zweiund dreiwertigen Eisen sowie dem Magnesium und vertritt sie wahrscheinlich in den Gittern der Pyroxene, der Hornblende, des Biotits und des Titanomagnetits, die nach der Tab. 11 ko- 
balthaltig sind. Der Kobaltgehalt dieser Mineralien ist jedoch kleiner als der Totalgehalt der Gesteine, woraus man schliessen kann, dass das Kobalt anders als das Nickel auch mit der Sulfidphase verbunden ist.

\section{Kupfer}

Die Anzahl der ausgeführten Totalanalysen des Kupfers ist 40. Die Analysen sind von denselben Proben wie die des Nickels und des Kobalts gemacht worden.

Auffallend ist das Schwanken des Cu-Gehalts in den analysierten Proben. In den Uralitdiabasen variiert der Kupfergehalt von $0.008 \%$ bis $0.026 \%$ und in den Grünsteindiabasen von $0.009 \%$ bis $0.07 \%$. Im Durchschnitt sind die Grünsteindiabase kupferhaltiger als die Uralitdiabase. Der durchschnittliche Cu-Gehalt der Uralitdiabase beträgt $0.016 \%$ und der der Grünsteindiabase $0.028 \%$.

Die Gesteine der spilitischen Differentiationsserie sind, was die Menge des Kupfers betrifft, den Metadiabasen ähnlich: Der Cu-Gehalt wechselt stark von Probe zu Probe. Es ist jedoch festzustellen, dass die durchschnittlichen Werte nach den albitreichen Typen zu zunehmen, wie es in der Tabelle $10 \mathrm{zu}$ sehen ist. In den Periotiten ist der Kupfergehalt am kleinsten, d.h. $0.010 \%$. Bei den hornblendereichen Albitdiabasen wächst er auf $0.030 \%$ und bei den albitreichen Albitdiabasen auf $0.027 \%$. Die Albitpegmatite und die Albitkarbonatgänge führen dagegen kein Kupfer in bedeutender Menge; in den kieshaltigen Quarz-Karbonatgängen kommt es relativ reichlich vor.

Das Kupfer ähnelt in Hinsicht auf seine Iongrösse $\left(\mathrm{Cu}^{2+}=0.72 \AA\right)$ dem Ferroeisen und ersetzt es in den Pyroxenen, in der Hornblende, dem Biotit und Titanomagnetit (s. Tab. 11). Nach Wager \& Mitchell (1951) kann auch der Plagioklas bemerkenswert kupferhaltig sein. Es ist nicht untersucht worden, in welchem Masse das Kupfer in den betreffenden Gesteinen im Plagioklas eingebaut vorkommt.
Der Cu-Gehalt der anderen Silikate beträgt $0.01 \%$ und dabei ist kein Schwanken unter den verschiedenen Differentiaten zu bemerken, obgleich der Totalgehalt des Kupfers variiert. Im allgemeinen ist der $\mathrm{Cu}-$ Gehalt der Silikate kleiner als der totale Kupfergehalt des Gesteins, was zeigt, dass ein bedeutender Teil des Kupfers mit dem Schwefel verbunden ist. In den Gesteinen sind auch Kupferkies, Bornit und Kupferglanz identifiziert worden.

Es soll hier vergleichsweise auch der durchschnittliche Kupfergehalt der Basalte erwähnt werden, der nach Wedepohl (1961) $0.0067 \%$, nach Vinogradov (1962) $0.010 \%$ und Agiorgitis (1968) $0.0067 \%$ beträgt. z.B. geben Markhinin \& Sapoznikova (1962) den Durchschnittsgehalt für Basalte der Kurilen mit $0.0063 \%$ an, und Dekkabasalte enthalten nach Sinha \& Karkare (1964) $0.0080 \%$ Cu. Mit Rücksicht auf den durchschnittlichen Kupfergehalt der Basalte lässt sich feststellen, dass die basischen Ergussgesteine des Koli-Kaltimogebiets sehr reich an Kupfer sind. Auffallend kupferreich sind die Grünsteindiabase und vor allem die albitreichen Albitdiabase.

\section{Schwefelisotopverhältnisse in der Sulfidphase}

In den letzten Jahren sind viele Untersuchungen über die Verteilung der Schwefelisotope in verschiedenen Formationen ausgeführt worden. Die geleistete Arbeit scheint sich zu einer vortrefflichen Hilfe für die Erklärung der Erzbildung zu entwickeln. Das ist der Fall vor allem in bezug auf die Frage, ob der Schwefel juvenil ist, oder ob er an dem grossen Stoffkreislauf teilgenommen hat, wie die Untersuchungen von Jensen (1959), Ault (1959) Ault \& Kulp (1960) und Thode u.a. (1962) zeigen. Diese Untersuchungen dienen als Grundlage dieses Kapitels.

Von den Schwefelisotopen sind ${ }^{32} \mathrm{~S}$ und ${ }^{34} \mathrm{~S}$ betrachtet worden. Die Ergebnisse werden ge- 
TABELle 12.

Schwefelisotopenverhältnisse in den kieshaltigen Karbonat-Quarzgängen des Koli-Kaltimogebiets. Die ergebnisse sind im Verhältnis zu NBS-120-Standard, der ${ }^{32} \mathrm{~S} /{ }^{34} \mathrm{~S}-$ Wert 22.20 hat, angegeben.

\begin{tabular}{|c|c|c|c|c|}
\hline Probe & Lage & Mineral & $\delta^{34} \mathrm{~S} \%$ & ${ }^{32} \mathrm{~S} /{ }^{34} \mathrm{~S}$ \\
\hline $78 / 87$ & $\mathrm{x}=501.3, \mathrm{y}=6968.2$ (Risti- & 7 & & \\
\hline $52 / 65$ & monttu) $\ldots \ldots \ldots \ldots \ldots \ldots \ldots$ & Schwefelkies & +1.0 & 22.178 \\
\hline $\begin{array}{l}52 / 65 \\
76 \mathrm{~b} / 67\end{array}$ & $\begin{array}{l}x=498.4, y=6970.6 \quad \text { (Kyykkä) } \\
x=502.2, y=6979.5 \ldots \ldots \ldots\end{array}$ & $\begin{array}{l}\text { Kupferkies } \\
\text { Kupferkies }\end{array}$ & $\begin{array}{l}-1.4 \\
-1.2\end{array}$ & $\begin{array}{l}22.231 \\
22.227\end{array}$ \\
\hline $75 \mathrm{~b} / 67$ & $x=497.5, y=6971.9$ (Hokka) & Kupferkies & -2.0 & 22.244 \\
\hline $75 b / 67$ & $\mathrm{x}=497.5, \mathrm{y}=6971.9$ (Hokka) & Bleiglanz & -2.3 & 22.251 \\
\hline
\end{tabular}

wöhnlich im Verhältnis der genannten Isotope oder als $\delta^{34} \mathrm{~S}_{0} / 00$ dargelegt, der von der folgenden Formel definiert wird:

${ }^{34} \mathrm{~S} 0 / 00=\frac{\left({ }^{34} \mathrm{~S} /{ }^{32} \mathrm{~S}\right) \text { Probe }-\left({ }^{34} \mathrm{~S} /{ }^{22} \mathrm{~S}\right) \text { Standard }}{\left({ }^{34} \mathrm{~S} /{ }^{22} \mathrm{~S}\right) \text { Standard }} \times 1000$

Zum Ausganspunkt für die Betrachtung wird das Schwefelisotopverhältnis ${ }^{32} \mathrm{~S} /{ }^{4} \mathrm{~S}$ der $\mathrm{Me}$ teorite genommen, das nahe der Kostante 22.20 liegt. Es ist festgestellt worden, dass die Schwefelisotopverhältnisse der Formationen, die ihrem Ursprung nach deutlich juvenil sind, sich dieser Konstante nähern. Der Schwefel aber, der an dem grossen Stoffkreislauf teilgenommen hat, ist fraktioniert worden. Die biogenen und und hydrothermalen Formationen haben sich mit dem leichten Isotop angereichert, während die Sulfate enthaltenden Formationen reich an dem schweren Isotop geworden sind.

Die Schwefelisotopverhältnisse der Sulfidphase des Koli-Kaltimogebiets sind mit dem Massenspektrometer von L. F. Casabona Group Leader bestimmt worden. Die Analysen sind insgesamt für fünf verschiedene Proben demacht worden, von denen drei die von alters bekannten kieshaltigen Quarz-Karbonatgänge von Kyykkä und Hokka (beschr. von Saksala 1960), eine die in einer Bruchzone auftretende, kleine pechblendehaltige Schwefelkieslagerstätte von Ristimonttu (s. Piirainen 1968) und eine am östlichen Strand des Ylä-Paukkajanjärvi liegende kleine, kupferkiesreiche Quarz-Karbonatgänge vertreten. Der analysierte Schwefel ist in der Probe von Ristimonttu mit Schwefelkies, in den Proben von Kyykkä und dem östlichen Strand des YläPaukkajanjärvi mit Kupferkies, und in den Proben von Hokka mit Kupferkies und Bleiglanz verbunden gewesen. Die Analysendaten sind in der Tabelle 12 zu sehen.

Die Analysen zeigen, dass die Isotopverhältnisse der Sulfidphase in den verschiedenen Teilen des Gebiets nahe der Schwefelisotopkonstante der Meteorite liegen, was darauf hinweist, dass der Schwefel juvelinen Ursprungs ist. Die Erdruste ist im Anfang des initialen Magmatismus kalt und starr gewesen, so dass keine Kontaminationserscheinungen haben stattfinden können. Die kleinen Abweichungen nach beiden Seiten der Konstante hin sind auf eine Fraktionierung zurückzuführen: Der Schwefelkies hat sich in geringem Masse mit dem schweren Isotop und der Kupferkies und mehr noch der Bleiglanz mit dem leichten Isotop angereichert. Die Kristallisationsfolge der genannten Mineralien ist nach Schneiderhöhn (1955) folgende: Schwefelkies, Kupferkies und Bleiglanz. Die Fraktionierung nach der Kristallisationfolge verstärkt die Auffasung, dass der Schwefel ursprünglich juvenil war.

Im nordkarelischen Schiefergebiet bieten die Erzlagerstätte von Outokumpu und die nahe liegenden Schwarzschiefer, deren schwefelisotopische Zusammensetzung von Kouvo \& Kulp (1961) untersucht worden sind, einen sehr interessanten Vergleichspunkt. Es ist bemerkenswert, dass die Schwefelisotopverhältnisse der initialen Erstarrungsgesteine teilweise denjenigen der Lagerstätte von Outokumpu entsprechen, aber 
von den Werten der Schwarzschiefer abweichen, die sich mit den leichten Isotopen angereichert haben. Der Vergleich der Schwefelisotopverhältnisse der Outokumpuerze und der Gesteine des initialen Magmatismus stützt die Auffassung von Borchert (1954-1955), dass die Entstehung der Erzlagerstätte von Outokumpu mit dem initialen Magmatismus verbunden sei; und weiter lässt sich annehmen, dass Schwefel initialmagmatischen Ursprungs eine weite Verbreitung in dem nordkarelischen Schiefergebiet hat.

\section{Initialer Magmatismus und Erzbildung}

Viele mit dem initialen Magmatismus verbundenen Chrom-, Titaneisen- und Nickelmagnetkieslagerstätten aus den Differentiationsserien Peridotit-Norit-Anorthosit tholeitischen Ursprungs sind in verschiedenen Teilen der Erde bekannt. Deswegen ist die sich an die tholeitische Assoziation anschliessende Erzbildung ziemlich gut erforscht. Dagegen ist die Erzbildung der spilitischen Assoziation weniger bekannt, was darauf zurückzuführen ist, dass man die Gesteine der spilitischen Assoziation als Erzeugenisse der Metamorphose erklärt hat, und dass sie zum grössten Teil aus Vulkaniten bestehen, die nicht zur Untersuchung der Erzbildung geeignet sind. Zwar hat in den letzten Jahren vom petrologischen Gesichtspunkt ausgehende Untersuchung mit Rücksicht auf die Orogenese und die Entwicklung der betreffenden Formationen als Funktion des orogenen Zyklus festgestellt, dass die mit dem initialen Vulkanismus verknüpfte Erzbildung reichlicher und vielseitiger ist als man früher angenommen hat (s. Borchert 1957, Cissarz 1957, Amstutz 1959). Man hat z.B. viele Eisen- und Manganerzlagerstätten sowie Sulfidvorkommen für submarinexhalative Bildungen gehalten, die an den initialen Magmatismus angeschlossen sind. Zunächst hat man diese Erzbildung durch die oben dargestellten mikroskopisch-petrographischen und geochemischen Untersuchungen erläutern wollen. Das Koli-Kaltimogebiet eignet sich gut zu derartigen
Untersuchungen, denn die Gesteine der tholeitischen und spilitischen Assoziation des initialen Magmatismus sind hier als hypabyssische Gänge anzusehen, in denen die Erzbildung ihren Anfang genommen hat, aber wegen Zeitmangels unterbrochen worden ist.

Von den Elementen, deren Verteilung auf die spilitische hypabyssische Differentiationsserie hier betrachtet worden ist, bilden oxydische, aber einige auch karbonatische Erzlagerstätten $\mathrm{Cr}, \mathrm{Fe}, \mathrm{Ti}, \mathrm{V}$ und $\mathrm{Mn}$ in Verbindung mit der normalen Kalk-Alkalserie oder mit der tholeitischen Assoziation, wie sie in dieser Arbeit genannt worden ist, und zwar so, dass Chrom in den Peridotiten zum Chromit, Eisen, Titan und Vanadin zwischen den Noriten und den Anortositen zum Titanomagnetit kristallisieren, während Mangan in die hydrothermalen Lösungen getrieben wird. Das reichliche Vorkommen von Wasser und Kohlendioxyd in tholeitischem Magma hat die Entstehung der spilitischen Gesteinsassoziation im KoliKaltimogebiet verursacht. Die Erzbildung der betreffenden Elemente hat dabei ungefähr in gleicher Weise stattgefunden wie bei der Entstehung der tholeitischen Assoziation. Das Chrom hat sich in den Peridotiten angereichert, die Bildung des vanadinhaltigen Titanomagnetits hat am reichlichsten in den albitreichen Albitdiabasen stattgefunden, und das Mangan ist in die Karbonatgänge gewandert.

Die Bildung der Titaneisenerze und Eisenerze muss hier näher betrachtet werden. Der Titanomagnetit kommt in spilitischen Gesteinen im Gegensatz zu den Diabasen in Form idiomorpher Kristalle vor. Der Vanadingehalt sowie auch die Menge der Ilmenitlamellen sind am grössten in den Kristallen der hornblendereichen Albitdiabase. Bei den späteren Differentiaten ist der Vanadingehalt sowie auch die Menge des Titanomagnetits klein. In diesem Zusammenhang ist es wichtig, die liquidmagmatischen Eisenerzlagerstätten vom Kirunatyp sowie auch die Skarneisenerze ins Gedächtnis zurückzurufen. In beiden Fällen ist vorgeschlagen (s. Fischer 
1950, Piirainen \& Piispanen 1968), dass die Erzbildung durch die Liquation stattgefunden hat. Die Bedingungen für die Liquation des Oxydschmelzes vom Magma haben sich aus den Experimenten Fischers (1950) ergeben: 1. das Magma ist sehr reich an Alkalen, 2. das Magma enthält leichtflüchtige Bestandteile, 3. die Oxydationsstufe ist hoch. Mit Rücksicht auf die Entwicklung des initialen Magmatismus und auf die Zusammensetzung der Spilite lässt sich annehmen, dass bei der Kristallisation der Spilite die Forderungen, die Fischer auf Grund der Experimente von der Liquation des Oxydschmelzes vorgeschlagen hat, erfüllt sind. Eisenerzlagerstätten sind ja im Zusammenhang der spilitischen Assoziation gewöhnlich. Sie sind reich an Eisen, aber arm an Titan und Vanadin, was darauf hindeutet, dass die Liquation in den späteren Phasen stattgefunden hat, wobei das Titan und das Vanadin zum grössten Teil verbraucht sind. Wie oben festgestellt wurde, ist der Vanadingehalt am grössten in den hornblendereichen Albitdiabasen.

In Hinsicht auf die Entstehung der Sulfiderzlagerstätten sind die Unterschiede deutlicher als hinsichtlich der Entstehung der oxydischen Erze zwischen den spilitischen und tholeitischen Assoziationen. Nach Schneiderhöhn (1958) entstehen die Nickel-Magnetkieserze aus dem tholeitischen Magma kurz nach der Kristallisation der Peridotite durch die Liquation, die die Abnahme des Magnesiums und Eisens und die Zunahme des Siliziumoxyds verursacht hat. Weiter verstärkt nach Schneiderhöhn das
Vorhandensein des Wassers im Magma dessen Fähigkeit, Sulfide zu lösen. So wird die Ausscheidung der Sulfidphase in dem spilitischen Magma im Vergleich zu dem normalen tholeitischen Magma bedeutend verspätet. Da nun die Tarnung des Nickels in den Silikaten bei hoher Temperatur kristallisierter Fraktionen grösser als diejenige des Cobalts ist, wird das Restmagma reicher an Kobalt als an Nickel. Die Tarnung des Kupfers in den Silikaten bleibt dagegen während der Kristallisation konstant. Wenn die Ausscheidung der Sulfidphase stattfindet, enthält diese Phase ausser Eisen und Kupfer im Vergleich zum Nickel, auch Kobalt in bedeutender Menge, sogar so viel, dass während der Kristallisation der Sulfidphase statt des Pentlandits Kobaltpentlandit entsteht. So enthält die mit der spilitischen Assoziation verknüpfte Sulfiderzeparagenese der höchsten Temperatur eine Schwefelkies-Magnetkies-KobaltpentlanditKupferkiesparagenese anstatt der in normaler tholeitischer Assoziation auftretenden Magnetkies-Pentlandit-Kupferkiesparagenese. Die Mineralparagenese ist auch in dem Felsgrund weit verbreitet, der aus der evolutionärtransgressiven Sedimentserie und den Gesteinen des initialen Magmatismus besteht, und sie kann sowohl intrusiv- als extrusiv-magmatische sowie sogar aus untermeerischen, vulkanischen Aushauchungen abgeschiedene Erzlagerstätten bilden. Gerade dieser Typ ist, wahrscheinlich durch die weitverbreiteten Kupfererzlagerstätten vom Outokumpu-Typ, im nordkarelischen Schiefergebiet vertreten.

\section{LITERATURVERZEICHNIS}

Agrongitis, G. (1968) Zur Geochemie einiger seltener Elemente in basaltischen Gesteinen. Tscherm. Min. Petr. Mitt., 12, S. 204-229.

Amstutz, G. C. (1959) Syngenese und Epigenese in Petrographie und Lagerstättenkunde. Schweiz. Min. Petr. Mitt. 39, S. $1-84$.

Aubourn, J. (1965) Geosynclines, Developments in geotectonics 1. Elsevier, Amsterdam.
Ault, W. U. (1959) Isotopic fractionation of sulfur in geochemical processes. Pp. 241-259 in Researches in geochemistry, edited by P. H. Abelson, John Wiley \& Sons, Inc., New York.

Ault, W. U., and Kulp, J. L. (1959) Isotopic geochemistry of sulfur. Geochim. Cosmochim. Acta 16 , pp. $201-235$.

Borchert, H. (1954) Kritische Anmerkungen zu zwei 
neuen Arbeiten über Outokumpu. Chemie der Erde 17 , S. $1-5$.

Borchert, H. (1956) Der initiale Magmatismus und die zugehörigen Lagerstätten. N. Jb. Miner. Abh. 91, Festband Schneiderhöhn, S. 541-572.

Carr, M. H., and Turekian, K. K. (1961) The geochemistry of cobalt. Geochim. Cosmochim. Acta 23 , pp. $9-60$.

Cissarz, A. (1956) Lagerstätten des Geosynklinalvulkanismus in den Dinariden und ihre Bedeutung für die geosynklinale Lagerstättenbildung. N. Jb. Miner. Abh. 91, Festband Schneiderhöhn S. 485-540.

Fischer, R. (1950) Entmischungen in Schmelzen aus Schwermetalloxyden, Silikaten und Phosphaten. N. Jb. Miner. Abh. 81, S. 315-364.

Frosterus, B. (1902) Bergbyggnaden i sydöstra Finland. Deutsches Referat: Der Gesteinsaufbau des südöstlichen Finnland. Bull. Comm. Géol. Finlande 13.

Frosterus, B. ja Wilkman, W. W. (1920) Vuorilajikartan selitys, lehti D 3, Joensuu. Suomen geologinen yleiskartta, 1: 400 000. Geologinen toimikunta, Helsinki. (Résumé en francais: In schwedischer Auflage).

GAÁL, G. (1964) Jatul und karelische Molasse im S-Koligebiet in Nordkarelien und ihre Beziehungen zum Gebirgsbau des präkambrischen Orogens. Bull. Comm. Géol. Finlande 213.

Goldschmidt, V. M. (1954) Geochemistry. Oxford, Clarendon Press.

Jensen, M. L. (1959) Sulfur isotopes and hydrothermal mineral deposits. Econ. Geol. 54, pp. 374-394.

Kennedy, W. Q. (1933) Trends of differentiation in basaltic magmas. Am. J. Sci. 25, pp. 239-256

Kouvo, O., and Kulp, J. L. (1961) Isotopic composition of Finnish galenas. Ann. N. Y. Acad. Sci. 91, pp. $476-491$.

Markhinin, E. K., and Sapozhnikova, A. M. (1962) Content of $\mathrm{Ni}, \mathrm{Co}, \mathrm{Cr}, \mathrm{V}$, and $\mathrm{Cu}$ in volcanic rocks Kamchatka and the Kurile Islands. Geochimiya 4, pp. $372-376$.

Meriläinen, K. (1961) Albite diabases and albitites in Enontekiö and Kittilä, Finland. Bull. Comm. Géol. Finlande 195.

Pirrainen, T. A. (1968) Die Petrologie und die Uranlagerstätten des Koli-Kaltimogebiets im finnischen Nordkarelien. Bull. Comm. Géol. Finlande 237.

Piirainen, T. A., and Piispanen R. (1967) On the origin of primary skarn iron ores. Bull. Comm. Géol. Finlande 229.

SAKSElA, M. (1960) Beiträge zur Kenntnis der sog. chloritischen Kupferformationen im fennoskandischen Grundgebirge. N. Jb. Miner. Abh. 94, Festband Ramdohr, S. 319-351
SCHNEIDERHÖHN， H. (1955) Erzlagerstätten, Kurzvorlesungen zur Einführung und zur Wiederholung. 3 Aufl., Gustav Fischer-Verlag, Stuttgart.

- (1958) Die Erzlagerstätten der Erde Bd. 1. Die Erzlagerstätten der Frühkristallisation. Gustav Fischer-Verlag, Stuttgart.

Sinha, R. C., and Karkare, S. G. (1964) Study of the distribution and behaviour of trace elements in some of the Deccan Basalts. Bull. Geol. Soc. of India 1.

Strlle, H. (1938) Die Grossfelder der Erdkruste und ihr Magmatismus (kurzes Vortragsprotokoll), Sitzungsber. d. Preuss. Akad. d. Wiss., Phys. - math. Kl. $51-52$.

- (1939) Zur Frage der Herkunft der Magmen. Abh. d. Preuss. Akad. d. Wiss., Math. - naturwiss. Kl., 19, Berlin 1940.

Thone, H. G., Dunford, B., and Shima, M. (1962) Sulfur isotopic abundances in rocks of Sudbury district and their geologic significance. Econ. Geol. 57 , pp. $565-578$.

Turekian, K. K., and Carr, M. H. (1960) The geochemistry of chromium, cobalt and nickel. Int. Geol. Congress, XXI Session, Norden, Pt. 1, S. 14-26.

Vinogradov, A. P. (1956) Regularities of distribution of chemical elements in the Earth's crust. Geochimiya $1,43$.

V̈̈YRYNEN, H. (1928) Geologische und petrographische Untersuchungen im Kainuugebiet. Bull. Comm. Géol. Finlande 78 .

- (1933) Über die Stratigraphie der karelischen Formationen. C. R. Soc. Géol. Finlande 6, S. 54. Bull. Comm. Géol. Finlande 101, S. 54.

- (1939) On the geology and tectonics of the Outokumpu ore field and region. Bull. Comm. Géol. Finlande, 124.

- (1954) Suomen kallioperä, Otava, Helsinki.

WAger, L. R., and Mrtchell, R. L. (1951) The distribution of trace elements during strong fractionation of basic magma - a further study of the Skaergaard Intrusion, East Greenland. Geochim. Cosmochim. Acta, S. 129-208.

Walker, F., and PoldervaArt, A. (1949) Karroo dolerites of the Union of South Africa. Geol. Soc. Amer. Bull. 60, pp. 591-706.

WeDEPOHL, K.H. (1961) Geochemische und petrographische Untersuchungen an einigen jungen Eruptivgesteinen NW-Deutschlands.

Fortschr. Mineral. 29, S. $142-148$.

Wegmann, C. E. (1928) Über die Tektonik der jüngeren Faltung in Ostfinnland. Fennia 50, 16.

Manuskript eingegangen am 16. August 1968. 